\title{
Casein Kinase 1 dynamics underlie the PER2 circadian phosphoswitch
}

10 Jonathan M. Philpott ${ }^{1 \dagger}$, Rajesh Narasimamurthy ${ }^{2 \dagger}$, Clarisse G. Ricci ${ }^{3 \dagger}$, Alfred M. Freeberg ${ }^{1}$, Sabrina R. Hunt ${ }^{1}$, Lauren E. Yee ${ }^{1}$, Rebecca S. Pelofsky ${ }^{1}$, Sarvind Tripathi ${ }^{1}$, David M. Virshup ${ }^{2,4 *}$, Carrie L. Partch ${ }^{1,5 *}$

15 'Department of Chemistry and Biochemistry, University of California Santa Cruz, Santa Cruz, CA 95064

${ }^{2}$ Program in Cancer and Stem Cell Biology, Duke-NUS Medical School, Singapore 169857

${ }^{3}$ Department of Chemistry and Biochemistry, University of California San Diego, La

20 Jolla, CA 92093

${ }^{4}$ Department of Pediatrics, Duke University Medical Center, Durham, NC 27710

${ }^{5}$ Center for Circadian Biology, University of California San Diego, La Jolla, CA 92093

${ }^{\dagger}$ Equal contributions

25

*Correspondence: cpartch@ucsc.edu, david.virshup@duke-nus.edu.sg

30

35

40 


\section{Summary}

Post-translational control of PERIOD stability by Casein Kinase $1 \delta$ and $\varepsilon$ (CK1) plays a key regulatory role in metazoan circadian rhythms. Despite the deep evolutionary conservation of CK1 in eukaryotes, little is known about its regulation and the factors that influence substrate selectivity on functionally antagonistic sites in PERIOD that directly control circadian period. Here we describe a molecular switch involving a highly conserved anion binding site in CK1. This switch controls conformation of the activation loop to define substrate selectivity on mammalian PER2, thereby directly regulating its

55 stability. Integrated experimental and computational studies shed light on the allosteric linkage between two anion binding sites that dynamically regulate kinase activity. We show that period-altering kinase mutations from humans to Drosophila differentially modulate this activation loop switch to elicit predictable changes in PER2 stability, providing a foundation to understand and further manipulate CK1 regulation of circadian rhythms.

\section{Keywords}

Circadian rhythms, Casein Kinase 1, phosphoswitch, activation loop, substrate recognition, non-consensus, conformational switch, Gaussian accelerated molecular dynamics, allostery, anion binding 


\section{Introduction}

Circadian rhythms are generated by a set of interlocked transcription/translation feedback loops that elicit daily oscillations in gene expression to confer temporal regulation to behavior, metabolism, DNA repair and more (Bass and Lazar, 2016). The PERIOD proteins (PER1 and PER2) nucleate assembly of large, multimeric complexes with the circadian repressors CRY1 and CRY2 that directly bind to and inhibit the core circadian transcription factor, CLOCK:BMAL1, on a daily basis (Aryal et al., 2017; Michael et al., 2017; Xu et al., 2015). PERs are stoichiometrically limiting for the assembly of these essential repressive complexes (Lee et al., 2011b). In this way, their abundance and post-translational modification state relay important biochemical information on the relative timing of the clock to other core clock proteins. Therefore, the expression, modification, and protein stability of PER1 and PER2 is under particularly tight regulation.

While both transcriptional and post-transcriptional mechanisms feature importantly in the rhythmic generation of PER proteins (Kojima et al., 2011; Takahashi, 2017), much attention has been focused on the post-translational control of PER stability

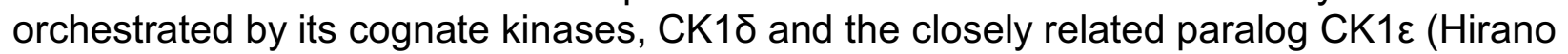
et al., 2016). These clock-associated kinases are somewhat unusual, in that they remain stably anchored to PER1 and PER2 throughout the circadian cycle (Aryal et al., 2017; Lee et al., 2001) via a conserved Casein Kinase Binding Domain (CKBD) (Eide et

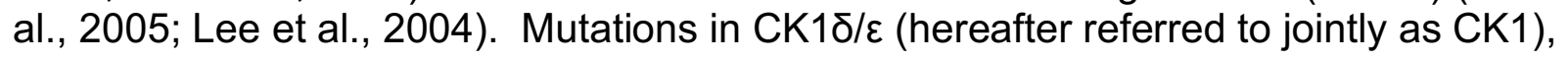
as well as PER2, exert powerful control over circadian period, altering the intrinsic timing of circadian rhythms by hours in vivo (Lowrey et al., 2000; Toh et al., 2001; Xu et al., 2005; Xu et al., 2007). Because circadian period is linked to the timing of sleep onset, PER2 or CK1-dependent alterations to human circadian period manifest as sleep phase disorders that influence behavior and wellbeing on a daily basis (Jones et al., 2013).

PER2 is regulated by a CK1-dependent phosphoswitch, where kinase activity at two antagonistic sites functionally interact to control PER2 stability (Zhou et al., 2015). Two features define the CK1 phosphoswitch: degradation is initiated by phosphorylation of a Degron located several hundred residues upstream of the CKBD to recruit the E3

105 ubiquitin ligase, $\beta$-TrCP (Eide et al., 2005; Vanselow et al., 2006); this is counteracted by sequential phosphorylation of five serines embedded within the CBKD known as the FASP region (Narasimamurthy et al., 2018). This region is named for a Ser to Gly polymorphism in human PER2 that disrupts this stabilizing multi-site phosphorylation, shortens circadian period, and leads to Familial Advanced Sleep Phase Syndrome (Toh 110 et al., 2001). Mutation of the Degron phosphorylation site has the opposite effect, stabilizing PER2 to significantly compromise circadian rhythms (Reischl et al., 2007) in a manner similar to its constitutive overexpression (Chen et al., 2009). Therefore, the balance of stabilizing and degrading phosphorylation by CK1 leads to a complex temporal pattern of degradation in PER2 that is important for circadian timing (Zhou et 115 al., 2015). 
Despite the importance of $\mathrm{CK} 1$ for circadian timing in eukaryotic organisms from humans to Drosophila, Neurospora, and green algae (Gorl et al., 2001; Kloss et al., 1998; van Ooijen et al., 2013; Xu et al., 2005), little is known about how its activity is regulated on clock protein substrates. CK1 is thought of as an anion- or phosphatedirected kinase, using negative charge on the substrate to template activity, for example, on a pSxxS consensus motif (Flotow et al., 1990). Biochemical studies exploring CK1 activity have primarily relied on the non-physiological acidic substrates casein (Venerando et al., 2014) and phosvitin (Lowrey et al., 2000), or used peptides harboring anion- or phosphate-driven motifs (Isojima et al., 2009; Marin et al., 1994; Shinohara et al., 2017). However, in vitro studies of clock-relevant kinase mutants using these non-physiological substrates have led to the puzzling conclusion that CK1 mutants that decrease or increase period length all have reduced kinase activity (Kivimae et al., 2008; Venkatesan et al., 2019).

This paradoxical observation motivated us to explore the molecular basis of CK1 activity on native PER2 substrates both in vitro and in cellular assays. To do this, we leveraged a comparative approach, examining multiple circadian mutants with a combination of cell-based, in vitro, structural and molecular dynamics methods. We discovered that the CK1 tau mutant (R178C) has reduced activity on the non-consensus priming of the FASP region as well as the downstream consensus sites, but exhibits a gain of function on the Degron site both in vitro and in cells (Gallego et al., 2006). Therefore, tau inverts PER2 substrate selectivity relative to the wild-type kinase to promote degradation of PER2. A mechanism for inverted substrate selectivity was suggested by the crystal

140 structure of the tau kinase domain, which demonstrated the presence of a two-state conformational switch in the activation loop. Anion binding at a pocket near the activation loop biases the switch towards one conformation that correlates with a preference for the FASP substrate, while mutations that disfavor anion binding favor the alternate conformation with enhanced activity towards the Degron. Molecular dynamics

145 studies reveal the conformation of the activation loop switch correlates with the substrate selectivity profile of WT and tau kinase. Specifically, we find the alternate conformation of the activation loop is stabilized in tau, forming the basis for its enhanced activity on the Degron. A comprehensive analysis of other short period kinase mutants from Drosophila to humans finds that they too differentially bias this intrinsic switch in 150 substrate selectivity to enhance phosphorylation of the Degron and turnover of PER2. The anion-triggered activation loop switch may be a general mechanism regulating CK1 substrate selection.

\section{Results}

155 The tau mutant has decreased activity on the FASP region

The phosphoswitch model is predicated on CK1-dependent degradation of PER2 by phosphorylation of a $\beta$-TrCP-specific Degron upstream that is inhibited by stabilizing phosphorylation of the FASP region (Figure 1A). We recently demonstrated that CK1 primes FASP phosphorylation in a slow, rate-limiting step at serine 659 (mouse PER2 160 numbering, Figure 1B), with phosphorylation of the downstream consensus sites following rapidly in a sequential manner (dashed arrows at pSxxS consensus, Figure 1B) (Narasimamurthy et al., 2018). The tau mutation (R178C) was originally identified 
as a missense mutation that spontaneously arose in Syrian hamster CK1E (Lowrey et al., 2000; Ralph and Menaker, 1988). Although tau enhanced Degron phosphorylation in cells to preferentially degrade PER2 (Gallego et al., 2006), the prevailing model was that a loss of function on the FASP region allowed for unchecked phosphorylation of the Degron. The mutation eliminates a positively charged residue in the first of three CK1 family-specific pockets (Sites 1, 2, and 3) that coordinate anions in the crystallographic structure (Figure 1C) (Longenecker et al., 1996). The Site 1 pocket where R178 sits is located adjacent to the active site and has been postulated to bind the phosphorylated priming site to position serines of the consensus motif at the active site (Longenecker et al., 1996; Zeringo and Bellizzi, 2014). Based on this model, it is predicted that tau should preferentially disrupt phosphorylation of the downstream consensus sites in the FASP region due to its inability to recruit the primed substrate.

To test this idea, we used a FASP peptide based on the native mouse PER2 sequence (Figure 1B) that was primed synthetically by phosphorylation at $\mathrm{S659}$. We also used a constitutively active version of the isolated wild-type (WT) or tau (R178C) kinase domain

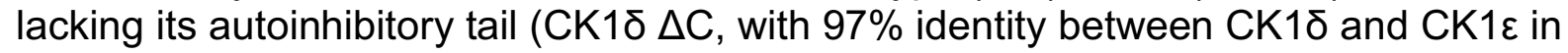
the kinase domain). As expected, tau had significantly lower activity than the WT kinase on this primed substrate (Figure 1D-E). This was also true for the minimal, primed synthetic substrate CK1tide (Figure S1) (Shinohara et al., 2017). We then asked if tau could influence priming phosphorylation using an unmodified FASP peptide. As we observed before with WT kinase, phosphorylation of the non-consensus priming site occurs with much slower kinetics than the downstream consensus sites (Figure 1F-G) (Narasimamurthy et al., 2018). To our surprise, we found that tau also had significantly diminished activity on an unprimed FASP substrate (Figure 1F-G), indicating that R178 is also important for the non-consensus priming event. This decrease in activity of tau on the priming site was also validated using an ELISA-based kinase assay with an antibody that is specific for phosphorylated S659 (Figure S1).

To confirm that tau influences both priming and downstream events at the FASP region, we used a real-time NMR-based kinase assay. In contrast to the traditional kinase assays above, this assay provides site-specific information about modification of the substrate by measuring the increasing volume of new peaks that arise for phosphorylated serines over time (Theillet et al., 2013). Because we already established the dependency of priming to initiate sequential phosphorylation of downstream sites in the FASP region by CK1 (Narasimamurthy et al., 2018), this assay should differentiate the effects of tau on non-consensus priming and phosphorylation of

200 the downstream consensus motif. If tau was simply deficient in recruitment of primed substrate, we should observe a similar degree of phosphorylation at the priming site (pS659) compared to WT kinase, but a decreased peak volume for the downstream serine (pS662). By contrast, we observed that the peaks for both pS659 and pS662 were both decreased in volume for tau (Figure $1 \mathrm{H}-\mathrm{I}$ ), with quantitative analysis of peak 205 intensities revealing that the ratio of consensus to priming activity (pS662/pS659) was similar in both WT CK1 and tau (Figure 1J). 
To test if these findings held in the context of full-length protein, we expressed mycPER2 and WT or tau myc-CK1ع in HEK293 cells and assessed phosphorylation using an anti-pS659-specific antibody. Consistent with our in vitro kinase assays, the activity of tau was much lower on the FASP priming site relative to the WT kinase (Figure 1K). As expected, phosphorylation of the subsequent serine was also decreased with tau (Figure S1). To examine the possibility that loss of activity on the FASP region was specifically due to the missense cysteine mutation in the original mutant (R178C), we also tested an R178A mutant in the cell-based assay and found that it also exhibited much lower activity on the FASP priming site in a cell-based assay (Figure S1). Collectively, these data show that tau is deficient in both the slow priming step as well as the downstream sequential, primed phosphorylation of the FASP region.

\section{tau exhibits a gain of function on the Degron}

Our previous model predicted that loss of function of tau for the FASP site allowed for unopposed phosphorylation of the Degron (Gallego et al., 2006). Using an antibody specific for phosphorylation of the CK1-dependent $\beta$-TrCP recruitment site at S478, we recapitulated the increased activity of tau on the Degron observed previously in myc-

225 PER2 that was transiently expressed with CK1 in HEK293 cells (Figure 1L) (Gallego et al., 2006). Consistent with the phosphoswitch model that FASP phosphorylation antagonizes CK1 activity on the Degron (Figure 1A), we observed an increase in activity of WT kinase on the Degron with the S659A mutant that abrogates priming and downstream phosphorylation of the FASP region (Figure 1L) (Narasimamurthy et al., 2018). We also found that tau had increased activity on the Degron in the S659A mutant, demonstrating that the tau mutant can still be regulated by the phosphoswitch. Given that kinase activity on the Degron is clearly linked to phosphorylation of the FASP in cells, we sought to clarify whether tau truly exhibits increased activity at the Degron using a peptide-based kinase assay in vitro (Figure $1 \mathrm{M}$ ). Here, we found that tau had significantly increased activity on the Degron relative to WT kinase (Figure $1 \mathrm{~N}-\mathrm{O}$ ).

These data suggest that in addition to any regulation imparted by the phospho-FASP on Degron activity in the context of full-length PER2, the tau mutation leads to a fundamental change in CK1 activity and substrate specificity.

\section{The tau mutation disrupts anion binding at Site 1 and Site 2 on CK1}

To explore the molecular basis for tau's altered substrate specificity, we solved a crystal structure of the CK1ס R178C kinase domain (Figure 2A and Table S1). Both WT and tau coordinate an anion at Site 3 similarly (Figure S2), but we observed a loss of anion binding at Sites 1 and 2 in tau. We expected that loss of the positively charged residue

245 at position R178 would disrupt anion binding at Site 1, although the mutation led to only minor structural changes in this anion binding pocket (Figure 2B). tau had an alternate conformation of the activation loop near the second anion binding pocket that clashes with binding of the anion (Figures $2 \mathrm{C}$ ), leading to its ejection from the site. This alternate conformation initiates at G175, three residues upstream of the tau mutation (Figure 2C).

250 A rotation of the backbone at G175 to a left-handed configuration dramatically alters the configuration of upstream residues to create a distinct conformation of the activation loop (Figures 2D and S2). A backbone flip of a glycine at this conserved position has been observed in other serine/threonine kinases, linking changes in conformation of the 
activation loop to regulation of kinase activity (Nolen et al., 2004); therefore, the 'loop up conformation observed in tau may lead to different kinase activity than the 'loop down' conformation observed in the WT kinase.

The activation loop is the key feature that distinguishes the CK1 family from other serine/threonine kinases. A deviation in sequence from the highly conserved 'APE'

260 motif in the $P+1$ region, which defines specificity for the residue that follows the phosphotarget, is largely attributed as the reason for a lack of mechanistic insight into CK1 substrate selectivity (Figure 2E) (Goldsmith et al., 2007). The activation loop and surrounding region also contain residues that coordinate the three anions observed in nearly all CK1 structures: the residues that coordinate binding at Sites 1 and 3 are

265 unique to the CK1 family, while R127 and K154, corresponding to Site 2, are broadly conserved in other kinase families. R127 is part of the highly conserved HRD motif that is used by some kinases to coordinate a phosphorylated residue in the activation loop and regulate substrate binding (Figure 2F) (Johnson et al., 1996). CK1 family kinases are generally considered to be constitutively active because they do not require 270 phosphorylation of the activation loop (Goldsmith et al., 2007). However, the activity of clock-relevant kinases CK1ס and CK1 $\varepsilon$ is inhibited by autophosphorylation of their disordered C-terminal tails (Graves and Roach, 1995; Rivers et al., 1998) and potentially by the phosphorylated FASP region (Figure $1 \mathrm{~L}$ ). Therefore, the anion binding sites could represent the basis for a CK1-specific regulatory mechanism by facilitating

275 the binding of phosphorylated C-terminal tails or substrates, and/or anionic signaling molecules (Fustin et al., 2018; Kawakami et al., 2008).

\section{Eliminating anion binding at Site 2 differentially regulates CK1 activity on the FASP and Degron}

280 Mutation of the residues corresponding to positions R127 and K154 at Site 2 severely decreases the activity of kinases that depend on phosphorylation of the activation loop (Gibbs and Zoller, 1991; Leon et al., 2001; Skamnaki et al., 1999). To test the role of Site 2 binding in regulating CK1 activity, we made charge reversion mutants at positions R127 and K154, as well as at K171 located nearby on the activation loop, and 285 measured enzymatic efficiency $\left(k_{\text {cat }} / K_{m}\right)$ on FASP and Degron peptides in vitro. We observed a modest decrease $(\sim 25 \%)$ in activity towards the FASP peptide (Figure S2 and Table S2), suggesting that the 'loop down' conformation that is enforced by anion binding at Site 2 may be important for FASP activity. Strikingly, the same mutations increased activity towards the Degron, with a $~ 50 \%$ gain in efficiency for R127E (Figure

290 S2 and Figure 2G). To further examine the role of K171 in regulation of anion binding, we solved a structure of the K171E mutant crystallized in high sulfate conditions and found a full complement of three anions bound (Figure S2). In our structure, the N170 sidechain replaced the mutant E171 to make an interaction with the sulfate, demonstrating that local flexibility in the activation loop allows it to retain sulfate binding 295 at Site 2 to some degree. The kinetic data suggest that variability in the strength of anion binding at the Site 2 pocket correlates with kinase activity on the FASP and Degron peptides. Notably, a decrease in activity on the stabilizing FASP region and an increase in activity on the Degron makes the Site 2 coordination mutants much more tau-like (Figure $2 \mathrm{H}$ ). This is consistent with a recent report that both tau and charge 
300 reversion mutants at Site 2 lead to decreases in PER2::LUC stability in cell-based assays (Shinohara et al., 2017).

\section{The activation loop switch is intrinsic to the CK1 family of kinases}

In looking at both copies of the kinase found in the asymmetric unit of the tau crystal, we discovered that the mutant kinase can take on either the 'loop down' or the 'loop up' conformation (Figure 3A). The activation loop is not stabilized by crystal contacts in the alternate 'loop up' conformation and was explicitly modeled based on good density in both conformations (Figure S3), suggesting that the kinase has an intrinsic ability to take on two discrete conformations in a switch-like manner. Since the first structure of

310 CK1 $1 \delta$ published over two decades ago, most have been determined after crystallization with high concentrations of sulfate or citrate anions (Long et al., 2012; Longenecker et al., 1996; Minzel et al., 2018). Because anion binding at Site 2 is incompatible with the activation loop in its 'loop up' conformation, prior crystallographic conditions have disfavored this alternate conformation. The WT structure that we used for our analysis

315 (PDB: 1CKJ) was first crystallized with a low concentration of anions, and then derivatized with tungstate as an analog for phosphate before data collection (Longenecker et al., 1996). Importantly, this structure also displays the same two discrete conformations marked by translocation of residue L173 (Figure 3A), confirming that the activation loop switch is an intrinsic property of the CK1ס kinase that has not

320 been explored functionally.

We then probed the library of existing CK1ס structures using the positioning of L173 as a quantitative metric for activation loop conformation by measuring interatomic distances of the L173 sidechain CD2 atom to either CD2 of L152 (short distance in the

325 'loop up' conformation, long in the 'loop down') or the hydroxyl of Y225 (long in the 'loop up', short in the 'loop down') (Figure 3B). A survey of 68 chains from 26 different crystal structures of CK1ס (in at least 7 space groups) demonstrated that tau and the WT kinase from PDB entry $1 \mathrm{CKJ}$ are the only structures of CK1 $\delta$ to have ever been captured in the 'loop up' conformation (Figure 3C). Moreover, the residues that 330 coordinate anion binding are broadly conserved in the CK1 family (Figure S6), and resident anions are observed in structures of other CK1 family kinases (e.g., CK1ع, CK1 33 ; Table S3), implicating anion binding and its regulation of the activation loop in a mechanism that may be generally conserved in the CK1 family. To determine if we could independently capture the two states of the activation loop switch in WT kinase,

335 we optimized sulfate-free crystallographic conditions and solved the structure of WT CK1ס. Here, similar to the 1CKJ WT structure, we also found both conformations of the activation loop in the two molecules of the asymmetric unit (Figure S3). Therefore, both conformations of activation loop switch can occur at low anion concentrations in WT kinase, but the tau mutation seems like it might favor the 'loop up' conformation

340 because it was observed in crystals that grew in the presence of high sulfate concentrations. Altogether, these data suggest that the tau mutation may allosterically regulate anion binding at Site 2 via the activation loop. 


\section{tau stabilizes the rare 'loop up' conformation of the CK1 activation loop}

345 To probe the dynamic behavior of WT CK1 and see how the enyzme is perturbed by the tau mutation, we performed Gaussian Accelerated Molecular Dynamics (GaMD) simulations (Miao et al., 2015) on four systems: WT and tau CK1 with the activation loop in the crystallographically-defined 'up' or 'down' conformations (Table S5). By monitoring the Root Mean Square Deviation (RMSD) of the activation loop with respect to the 'down' or 'up' crystallographic conformations, we set out to assess its stability over the course of 500 ns simulations (Figure 4A-D). We found that the activation loop remained stably in position when simulations were started from the 'loop down' conformation for both tau and WT. Similar results were seen when the anion was computationally removed from Site 2, suggesting that this conformation of the activation loop is intrinsically stable (Figure S4). However, in simulations starting from the 'loop up' conformation, the WT activation loop rapidly underwent a conformational change, as shown by increased RMSD up values. Because we did not see a concomitant decrease in the RMSD down values, we can conclude that this is not a complete transition from 'loop up' to 'loop down' on this timescale. Importantly, these transitions occurred more frequently in WT CK1 than in tau (Figure 4C-D). This confirms that the 'loop up' conformation is better tolerated in tau, consistent with our observation of this apparently rare conformation in our crystal structure (Figure 3).

\section{The activation loop allosterically controls the dynamics of loop L-EF in tau}

While monitoring the overall dynamics of WT and tau CK1, we detected a major difference in the dynamics of the loop connecting a-helices $E$ and $F$ (loop L-EF, Figure S4), which is part of the anion binding site disrupted by the tau mutation (Figure 4E-H). This is intriguing because temperature-dependent dynamics of loop L-EF were recently shown to be important for the temperature compensated activity of CK1 on PER2 (Shinohara et al., 2017). In our simulations of the WT kinase, this loop exhibited relatively restricted mobility, regardless of whether the activation loop was in the 'up' or 'down' conformation. Similar results were observed when the anion was computationally removed from Site 2 (Figure S4). However, we observed that the 'loop down' conformation of the activation loop in tau was accompanied by a strong disorganization of loop L-EF (Figure 4H), leading to significantly larger conformational freedom compared to the WT kinase (Figure 4G). Surprisingly, the enhanced dynamics of loop LEF was not observed when tau was in the 'loop up' conformation; instead, loop L-EF displayed the same restricted dynamics as the WT kinase (Figure E-F). These data suggest that the disruption of Site 1 by loop L-EF dynamics in the tau mutation may be due to allosteric communication with the activation loop, and by proxy, anion binding at Site 2.

\section{tau dynamically reshapes the substrate-binding cleft in CK1}

To investigate how the activation loop conformation and dynamics of loop L-EF might influence substrate selectivity, we calculated the volume of the substrate binding cleft and adjacent anion binding sites throughout the 500 ns GaMD simulations (Figure 4 and S4). As expected, Site 2 was only completely open only when the activation loop was in the 'loop down' conformation for the WT kinase (Figure 4I). Interestingly, this site became partially open in simulations of the WT kinase starting from the 'loop up' 
conformation (Figure 4J), indicating that the intermediate conformational state observed in our simulations might allow the kinase to recover, to some extent, the ability to bind an anion at Site 2. The conformational state of the activation loop indirectly affects the volume of the substrate binding cleft with opposing effects in WT and tau. In WT, the substrate binding cleft was open more consistently with the activation loop in its preferred 'down' conformation (Figure 4I-J). By contrast, the substrate binding cleft was open more consistently in tau in the 'loop up' conformation (Figure 4L) and, due to the dynamic disordering of loop L-EF, often closed when in the 'loop down' conformation (Figure 4K). Because the activation loop does not contact the substrate binding cleft, it cannot directly affect the shape of the cleft by steric effects. Instead, closing of the 400 substrate binding cleft in tau occurs due to the conformational disorganization in loop L$E F$, which is allosterically induced when the activation loop is in the 'down' conformation.

\section{tau influences the global dynamics of CK1}

405 We next used principal component analysis to uncover effects of tau on the principal modes of motion displayed by CK1 during the GaMD simulations (Figure 5, S5 and Supplemental Movie 1). The $1^{\text {st }}$ principal component consisted of a clear 'open-andclose' movement of the enzyme, achieved mainly by dislocation of the N-terminal lobe (N-lobe) with respect to the top of the helix $F$ (Figure 5A). This mode of motion has been

410 shown to control accessibility to the ATP-binding site and regulate substrate access in other kinases (McClendon et al., 2014). The histograms of the $1^{\text {st }}$ principal component show that WT CK1 sampled more of the open conformations compared to tau (Figure $5 \mathrm{C})$. The $2^{\text {nd }}$ principal component consisted of a twisting movement of the N-lobe with respect to the top of helix $F$ and significant rearrangement of loop L-EF, which can

415 either be extended or collapsed (Figure 5B). When collapsed, loop L-EF had the effect of sterically closing the substrate binding cleft. The histograms of the $2^{\text {nd }}$ PCA illustrate that loop L-EF adopts more extended conformations in WT CK1 and more collapsed conformations in tau (Figure 5D). The collapsed conformations require loop L-EF to undergo a significant conformational change, which agrees with the high conformational

420 freedom seen in this region when the tau activation loop is in the 'loop down' conformation. Altogether, the GaMD simulations suggest that the tau kinase never behaves fully like the WT enzyme; compared to WT, it stabilizes the rare, Degronpreferring conformation of the activation loop that remodels the substrate binding cleft and excludes anion binding at Site 2. By contrast, when tau samples the 'loop down' 425 conformation of the activation loop, it leads to a dynamic disordering of loop L-EF that favors conformations of the kinase that likely decrease its enzymatic efficiency on the FASP region.

\section{Circadian alleles from Drosophila to humans occur throughout CK1}

430 We mapped known mutant alleles that influence circadian rhythms onto the CK1 structure to gain further insight into its mechanism of regulation (Figure 6). Doubletime (DBT), the CK1 $1 \delta / \varepsilon$ ortholog in Drosophila, has one allele that causes a short circadian period $\left(d b t^{S}\right.$, P47S) while all others lead to a long period (Kloss et al., 1998; Rothenfluh et al., 2000; Suri et al., 2000). Many long period mutations occur at or near catalytically important residues. The classic $d b t^{L}$ allele M80I (Kloss et al., 1998) is directly adjacent 
to the catalytic lysine, K38; notably, expressing low levels of the catalytically dead K38R mutant in Drosophila also leads to a long period (Muskus et al., 2007). $d b t^{A R}$ and $d b t^{G}$ correspond to H126Y and R127H of the catalytically important HRD motif at Site 2 (Rothenfluh et al., 2000; Suri et al., 2000); together, these long period mutations sandwich the catalytic DFG motif and the regulatory spine that controls kinase activity (Figure 6B) (Taylor and Kornev, 2011). Two loss of function alleles occur in the activation loop: $d c 0^{18}(\mathrm{~S} 181 \mathrm{~F}$ ) is located right behind the tau site (R178), linking it to the substrate binding channel and activation loop, while $d c o^{2}$ (G175S) occurs at the hinge point for the activation loop switch (Zilian et al., 1999). Although Drosophila and mammalian PER proteins are somewhat functionally divergent, conservation of their Degrons (Chiu et al., 2008; Eide et al., 2005) and FASP-like stabilizing phosphorylation sites (Kivimae et al., 2008; Top et al., 2018; Xu et al., 2007) suggests that there may be some conservation in their regulation by CK1 (Figure 6C). In line with this, expressing mammalian $\mathrm{CK} 1 \delta$ with the tau or $d b t^{s}$ mutation leads to short period circadian rhythms

450 in flies (Fan et al., 2009). Moreover, there is an incredible degree of conservation in the CK1 family; the entire surface-exposed area linking Sites 1 and 2 and the substrate binding cleft are $\geq 95 \%$ identical in 20 species from humans to unicellular green alga where CK1 has been implicated in regulation of circadian clocks (Figure S6).

455 Other short period mutants exhibit differential activity on the FASP and Degron We were intrigued by three short period mutants from humans and Drosophila (T44A, $\mathrm{H} 46 \mathrm{R}$, and P47S) that colocalize in the N-lobe right above Site 2 (Figure 6B). Given the changes in $\mathrm{N}$ - to $\mathrm{C}$-lobe dynamics that we observed in our simulations of CK1 (Figure $5)$, we wondered how these mutations would influence substrate selectivity. We first 460 tested the activity of these short period kinase mutants in cell-based transfection assays by monitoring both FASP priming (Figure 7A) and Degron (Figure 7B) phosphorylation. Similar to the Site 2 mutants we tested earlier (Figure S2), the short period mutants T44A, H46R, and P47S each retained substantially more kinase activity on the FASP priming site than tau. A triple mutant of all three short period mutants (3M) did not have 465 additive effects. Likewise, they also appeared to retain kinase activity or even exhibited modest increases in Degron phosphorylation relative to WT (Figure 7B), although not to the same degree as tau. On balance, it appears that the Site 2 mutants (short period or anion binding) might act differently from tau in that they retain FASP priming activity while still increasing activity at the Degron relative to WT.

We also examined another short period mutant located at Site 1, K224D, which was recently reported to have a $\sim 20$-hour circadian period (Shinohara et al., 2017). Like the other short period mutants positioned near Site 2, K224D retained FASP priming activity (Figure 7C). However, in contrast to them, K224D exhibited a sharp increase in Degron 475 phosphorylation in HEK293 cells relative to WT that was similar to tau (Figure 7D). This effect was not dependent on the charge inversion, as we saw the same effect with a K224A mutant (Figure S7). Notably, our in vitro assessment of K224D enzyme efficiency showed an overall decrease in efficiency on the FASP peptide containing multiple serines (Table S2). Taken together, these data suggest that priming of the

480 FASP site S659 is intact, but subsequent downstream phosphorylation might be compromised in the mutant. To test this, we used the NMR-based kinase assay to 
provide single residue resolution on the stepwise phosphorylation of the FASP region (Narasimamurthy et al., 2018). Consistent with the cellular data, K224D retained normal priming activity at $\mathbf{S 6 5 9}$, but phosphorylation of the downstream serines that conform to the pSxxS consensus motif was compromised relative to WT kinase (Figure 7E). Just like blocking phosphorylation of the FASP region with the S659A mutant (Figure 1L), loss of phosphorylation at these downstream sites could allow for the increased kinase activity on the Degron that we observed in cellular assays. Therefore, although tau and K224D both enhance Degron phosphorylation to a similar degree and lead to 20-hour circadian periods (Lowrey et al., 2000; Shinohara et al., 2017), they likely achieve this through different mechanisms on the kinase_tau dynamically disrupts Site 1 in the FASP-preferring 'loop down' conformation of the activation loop to reduce both priming and downstream phosphorylation of the FASP region, while K224D (and K224A) likely just disrupt the anion binding at Site 1 that is important for phosphorylation of the pSxxS consensus motifs.

\section{The ratio of FASP/Degron enzyme efficiency correlates with PER2 stability}

One commonality of short period mutants should be enhanced Degron phosphorylation in cells, as both cellular (Gallego et al., 2006; Vanselow et al., 2006) and in vivo studies of short period mutants demonstrate that PER2 stability is decreased in a CK1dependent manner (Price et al., 1998; Xu et al., 2007). To test this model, we measured the effect of mutant CK1 co-expression on the half-life of PER2::LUC in real-time after cycloheximide treatment (Figure 7F and S7). All of the mutants, apart from L173A, lead to a shorter half-life of PER2::LUC (Figure 7G). We mutated L173 because it appeared to serve as a latch for the activation loop in both its 'loop up' and 'loop down' conformations (Figure $2 \mathrm{C}$ ). We found that the mutant substantially decreased kinase activity at both the FASP priming and Degron sites in the transfection-based assay and in vitro (Figure 7A-B and Table S2), and a double mutant with tau decreased its activity on the Degron to a similar degree (Figure S7). We hypothesized that the L173A mutant

510 might have a half-life similar to WT because its reduced phosphorylation of the stabilizing FASP region was offset by decreased activity on the Degron (Figure $7 G$ and Table S2). This suggested to us that the ratio of intrinsic kinase activity on the stabilizing FASP region relative to the Degron might account for CK1's ability to initiate PER2 degradation by $\beta$-TrCP (Eide et al., 2005; Vanselow et al., 2006). Indeed, Figure 7H

515 shows that a significant correlation exists between PER2::LUC half-life and the intrinsic ratio of CK1 kinase activity on these two key sites that establish the phosphoswitch mechanism. Taken with the other aspects of this study, our data strongly suggest that CK1 uses activation loop dynamics to orchestrate substrate specificity and contribute directly to the phosphoswitch regulation of PER2 stability, and therefore, the timing of 520 circadian rhythms (Figure 7I).

\section{Discussion}

Despite its powerful control over the timing of circadian rhythms along the entire branch of eukaryotes from humans to green algae (van Ooijen et al., 2013; Xu et al., 2005),

525 very little is known about the molecular determinants of CK1 $1 \delta$ substrate selectivity and activity. Here we identify a conformational switch in the activation loop of CK1 that regulates its activity on two distinct non-consensus motifs that underlie the 
phosphoswitch controlling PER2 expression and circadian timing in mammals (Zhou et al., 2015). Anion binding at Site 2 and the intrinsic dynamics of the conformational switch bias the WT kinase towards phosphorylation of the FASP region to stabilize PER2. We show that reducing anion binding at Site 2 regulates the activation loop to reshape the substrate binding cleft and enhance activity on the Degron. Using a series of mutations in the kinase that shorten circadian period from Drosophila to humans, we demonstrated that all exhibit enhanced activity on S478 in the Degron, consistent with our earlier study (Gallego et al., 2006). However, only the tau mutant has decreased activity on the FASP priming site and downstream serines. Molecular simulations revealed that enhanced dynamics of loop L-EF likely underlie the decreased activity of the tau mutant CK1 on the FASP region. Moreover, they also demonstrated that the activation loop switch can exert allosteric control over the dynamics and conformation of loop L-EF through the tau site.

Allosteric regulation is a common feature of protein kinases, often based on dynamic changes in ensembles of residues that can occur in the absence of major changes in conformation (Kornev and Taylor, 2015). CK1 has remarkable, histone-like

545 conservation ( $\geq 95 \%$ identity) of the entire surface-exposed area linking the two key anion binding sites and substrate binding cleft, suggesting that the mechanisms we discovered here likely apply broadly to circadian rhythms as well as other CK1regulated processes in other eukaryotes. Binding of regulatory anions at these conserved sites could arise from phosphorylated CK1 itself in cis via its autoinhibitory

550 tail (Rivers et al., 1998), or in trans from the binding of phosphorylated substrates like the FASP region, to allow for the generation of feedback regulation directly on the kinase. In line with this, changes in the sequence (Fustin et al., 2018) or

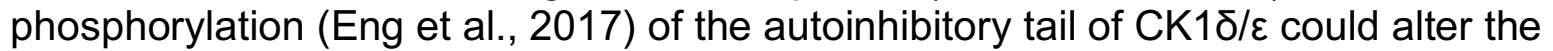
balance of FASP and Degron phosphorylation to control circadian period. In this way, 555 our study demonstrates that the dynamics of $\mathrm{CK} 1 \delta / \varepsilon$ directly encode its activity in the PER2 phosphoswitch (Zhou et al., 2015). The conformational equilibrium of the activation loop may also play a role in the temperature-compensated activity of $\mathrm{CK} 1 \delta / \varepsilon$ observed in vitro that is linked to the dynamics of loop L-EF, an insertion in the clockrelevant kinases $\mathrm{CK} 1 \delta$ and $\mathrm{CK} 1 \varepsilon$ that plays a key role in temperature compensation of 560 circadian rhythms (Shinohara et al., 2017).

We used PER2 stability here as a cellular proxy to study the effect of mutations from

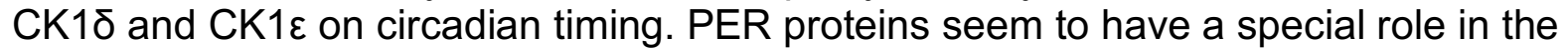
mammalian clock as state variables that define both the timing and phase of circadian

565 rhythms through changes in their abundance (Balsalobre et al., 2000; Zylka et al., 1998). CK1-dependent changes in PER abundance likely affect circadian period based on their role as stoichiometrically limiting factors in the assembly of transcriptional repressive complexes in the feedback loop of the molecular clock (Aryal et al., 2017; Kim and Forger, 2012; Lee et al., 2011b). Recent studies have shown that CK1 is 570 maintained in an active state while bound to PER2 in vitro (Qin et al., 2015), which may allow it to target other clock proteins for phosphorylation in the repressive complexes (Aryal et al., 2017), a property that seems to be conserved in Drosophila (Yu et al., 2006). Therefore, more studies are needed to fully understand the interplay between 
PER2 stability, PER-CK1 interactions and the regulation of post-translational modifications, including by phosphatases (Lee et al., 2011a) and other kinases (Hayasaka et al., 2017; Hirota and Kay, 2009; Oshima et al., 2019), that ultimately control circadian rhythms.

CK1 is highly active on primed (pSxxS) sites (Narasimamurthy et al., 2018), supporting its designation as the canonical consensus motif of the kinase. However, we find it compelling that many of the biologically important roles of $\mathrm{CK} 1 \delta / \varepsilon$ and the related kinase CK1a as key regulators of Wnt signaling (Marin et al., 2003), the DNA damage response (Knippschild et al., 1997), cell cycle (Penas et al., 2015), and circadian rhythms (Kloss et al., 1998; Lowrey et al., 2000) depend on their activity on non-

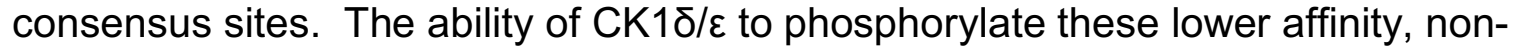
consensus sites on PER2 is likely dependent on the formation of a stable, stoichiometric complex with PER2 via two highly conserved sites that flank the FASP phosphorylation region in the CKBD (Eide et al., 2005; Lee et al., 2004). The generally low activity of CK1 that we observe on clock-relevant non-consensus sequences may also be important for the slow timescale of circadian rhythms. This property is also conserved in KaiC, the enzyme that controls circadian timing in the cyanobacterial system (Abe et al., 2015), suggesting commonalities in the biochemical origins of building slow biological clocks.

\section{Acknowledgments}

We would like to thank Danny Forger, Jae Kyoung Kim, Yinglong Miao, and J. Andrew McCammon for useful discussions and Sivakumar Parthiban for technical assistance. We also thank the beamline staff for their assistance at the Advanced Photon Source beamline 23-ID-D and Advanced Light Source beamline 8.3.1, as well as the San Diego Supercomputer Center (SDSC) for technical support. This work was funded by the National Medical Research Council of Singapore Grant NMRC/CIRG/1465/2017 (to D.M.V.) and National Institutes of Health Grants R01 GM031749 (to C.G.R.), GM107069 and R01 GM121507 (to C.L.P.), as well as funds from the NIH Office of the Director under Award S10 OD018455 for the $800 \mathrm{MHz}$ NMR spectrometer used here. S. Hunt was supported by NRSA F32 GM133149.

\section{Author contributions}

Conceptualization, J.M.P., R.N., C.G.R., D.M.V., and C.L.P.; Methodology, Investigation, J.M.P., R.N., C.G.R., A.M.F., S.R.H., L.Y., and R.P.; Validation, J.M.P.,

610 R.N., S.T.; Formal Analysis, J.M.P., R.N., C.G.R.; Visualization, J.M.P., R.N., C.G.R., and C.L.P.; Writing-Original Draft, J.M.P., C.G.R., and C.L.P.; Writing-Review and Editing, J.M.P., R.N., C.G.R., A.M.F, S.R.H., D.M.V., and C.L.P.; Supervision, D.M.V., and C.L.P.; Funding Acquisition, D.M.V., and C.L.P. All authors consented on the final draft of the manuscript.

\section{Declaration of interests}

The authors declare no competing interest. 


\section{Figure 1 tau alters CK1 substrate selectivity on PER2 to enhance Degron} phosphorylation

A, Domain map of PER2 with tandem PAS domains, Casein Kinase-binding domain (CKBD), CRY-binding domain (CBD) and CK1 phosphorylation sites. B, Sequence of the mouse PER2 FASP peptide with the priming site (S659, bold) and two downstream phosphorylation sites (S662 and S665, bold) that are phosphorylated sequentially by

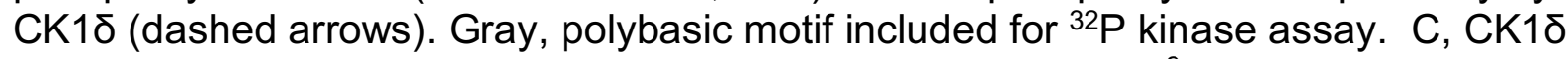
kinase domain with 3 anion binding sites (PDB: $1 \mathrm{CKJ}$ with $\mathrm{WO}_{4}{ }^{2-}$ anions). R178, blue. $\mathrm{D}$, Kinase assay with $20 \mathrm{nM}$ CK1ס $\Delta \mathrm{C}$ WT or tau on $200 \mu \mathrm{M}$ of primed FASP peptide (pS659) ( $n=4$ with s.d.). E, Phosphorylation rates on primed FASP ( $n=4$ with s.d.). Significance assessed by unpaired Student's two-sided t-test: ${ }^{*}, p<0.01$. F, Kinase assay as in D, but with $200 \mathrm{nM}$ CK1 $1 \delta \mathrm{C}$ WT or tau on $200 \mu \mathrm{M}$ of unprimed FASP $(\mathrm{n}=$ 4 with s.d.). G, Phosphorylation rates on the unprimed FASP peptide ( $n=4$ with s.d.).

635 Significance assessed as above. $\mathrm{H}$, Overlaid ${ }^{15} \mathrm{~N} /{ }^{1} \mathrm{H} H S Q C$ spectra at $3 \mathrm{hr}$ timepoint in the NMR kinase assay on $200 \mu \mathrm{M}{ }^{15} \mathrm{~N}$ FASP (black) $\pm 1 \mu \mathrm{M}$ WT (gray) or tau (red) CK1 $\triangle$ C. Arrows, phospho-specific peaks corresponding to pS659 and pS662. I, Phosphoserine peak intensities for pS659 and pS662 by WT and tau kinases from NMR kinase assay. J, Ratio of consensus to priming activity on the FASP (pS662/pS659) in

640 the NMR kinase assay. Errors were estimated from the standard deviation of the noise in the spectrum. K-L, Western blot of FASP priming site, detecting pS659 (K) or the Degron, detecting pS478 (L) on mouse myc-PER2 in HEK293 cell lysates after transfection with indicated expression plasmids. Representative blot from $n=3$ shown. Wedge, 10 or $50 \mathrm{ng}$ of myc-CK1 1 plasmid used. *, non-specific band. M, Sequence of 645 mouse PER2 Degron peptide with S478 (bold) and polybasic motif (gray). N, Kinase assay with $200 \mathrm{nM}$ kinase on $200 \mu \mathrm{M}$ Degron peptide ( $\mathrm{n}=4$ with s.d.). O, Phosphorylation rates on Degron ( $n=4$ with s.d.). Significance assessed as above. See also Figure S1.

650 Figure 2 tau alters anion binding on CK1ס

A, Overlay of WT kinase domain (gray, PDB: 1CKJ, chain B) with tau (maroon, PDB: $6 \mathrm{PXN}$, chain $\mathrm{A})$. The 3 anion binding sites $\left(\mathrm{WO}_{4}{ }^{2-}\right.$, from $\left.1 \mathrm{CKJ}\right)$ are labeled. $\mathrm{B}$, View of Site 1 in WT (top, gray) and tau (bottom, maroon). Polar interactions, dashed black lines. C, Overlaid view of Site 2 in WT and tau as above. Polar interactions, dashed black lines. Asterisk, hinge point for conformational change at G175. Note: Site 2 anion is only bound in WT, as it is blocked by the activation loop in tau. D, Representation depicting the left-hand configuration of G175 and subsequent rotation (solid arrow) of upstream residues T174 and L173. E, Alignment of the activation loop of CK1ס with representatives of other serine/threonine kinase families. Residues that coordinate anion binding on CK1 are indicated above in gray. F, Superposition of the Site 2 anion binding site in CK1 with the binding site for the phosphorylated activation loop of other serine/threonine kinases. Depicted are: PKB (PDB: 106K, pale cyan), PDK1 (1H1W, aquamarine), CDK2 (1QMZ, green cyan), ERK2 (2ERK, teal) and CK1ठ (5X17, dark gray). Residues that coordinate the anion are depicted in sticks, as are phosphoserine or phosphothreonine residues from other kinases; the $\mathrm{SO}_{4}{ }^{2-}$ coordinated at Site 2 by 
CK1ס (PDB: 5x17) is shown in transparent spheres. G, Enzymatic efficiency on the Degron ( $n=3$ with s.d.). Significance assessed relative to WT with an unpaired Student's two-sided t-test: ${ }^{* *}, p<0.01 ;{ }^{* * *}, p<0.001 ;{ }^{* * * *}, p<0.0001$. H, Ratio of enzymatic efficiency on FASP relative to Degron ( $n=3$ with s.d.). Equivalent activity on FASP and Degron, dashed line. Significance assessed as above. See also Figure S2.

Figure 3 tau alters an intrinsic molecular switch in the activation loop of CK1ס A, View of the activation loop switch in WT (PDB: 1CKJ, chains A (cyan) and B (gray)) and tau (PDB: 6PXN, chains A (maroon) and B (salmon)). B, The position of L173 CD2 relative to either $\mathrm{L} 152 \mathrm{CD} 2$ or $\mathrm{Y} 225 \mathrm{OH}$ reports on the conformation of the activation loop in the 'loop down' (gray) or 'loop up' (cyan) conformation in WT CK1ठ (PDB:

$1 \mathrm{CKJ}$ ). C, Scatter plot of interatomic distances in $\AA$ (from panel B) measured in 68 chains from 26 different crystal structures of CK1ס $\Delta C$. See Figure S3 and Supplemental Table 3 for more information.

\section{Figure 4 Probing the dynamics of CK1ס with GaMD simulations}

A-D, Stability of the activation loop assessed by the RMSD of residues 168-175 with respect to the 'loop down' (RMSD down, blue) or 'loop up' conformation (RMSD ${ }^{\text {up }}$, pink), as observed in the crystal structure. For each system, the RMSDs from all five MD

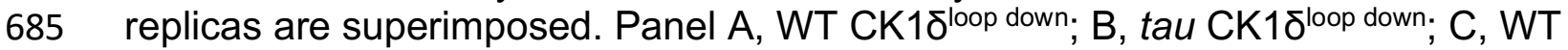

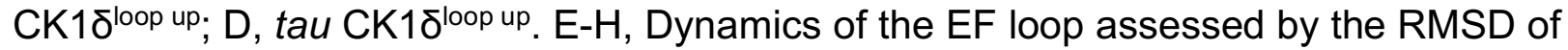
residues 213-224 with respect to the initial structure. For each system, the RMSD was calculated for individual replica (gray lines, $n=5$ ) and then averaged (green). The molecular representations in panels E-H show the crystallographic structure of CK1ס (gray) superimposed with snapshots of the L-EF loop extracted from the GaMD simulations (green). When present in the crystal structure and the simulation, sulfate anions are represented by spheres. I-L, Alterations in anion and substrate-binding clefts arise from the activation loop switch and tau mutation. Volumes for the binding clefts were extracted and averaged from GaMD simulations in the four states: panel I, WT

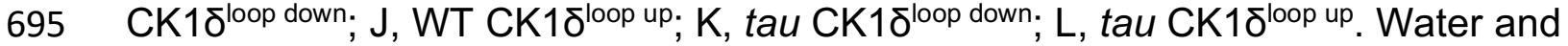
anions were removed from the analysis. Volumetric maps are contoured at 0.1 and represent regions that were consistently open during the simulations. See also Figure S4.

700 Figure 5 tau influences the principal modes of motion in CK1ס Principal modes of motion obtained from Principal Component Analysis (PCA). A, PC1 consists of an 'open-and-close' movement achieved by dislocation of the N-lobe with respect to the top of the helix $F$ to control accessibility of the ATP-binding site (dotted circle). B, PC2 consists of a twisting movement of $\mathrm{N}$-lobe with respect to the top of helix $705 \mathrm{~F}$ and significant rearrangement of the loop L-EF, which can be extended or collapsed against the substrate binding site (dotted circle). C-D, Structural representations correspond to (panel A) PC1 = -80 $\AA$ (cyan), PC1 = $80 \AA$ (blue) and (panel B) PC2 = -60 $\AA$ (orange), and PC2 $=60 \AA$ (red). The histograms represent projections of the accumulated GaMD trajectories along the $1^{\text {st }}(C)$ or $2^{\text {nd }}(D)$ principal components for WT 710 CK1ס and the tau mutant, either in the activation 'loop down' (black) or 'loop up' (white) conformations. See also Figure S5 and Supplemental Movie 1. 
Figure 6 Proximity of CK1 alleles map to catalytic and substrate binding sites A, Structure of CK1ס (PDB: 1CKJ, chain B) clock relevant alleles mapped from mammalian CK1 or Drosophila DBT. Mutants are colored by phenotype: short period (blue), long period (green), loss of function (gray). DFG catalytic motif, cyan. B, View of $\mathrm{N}$-lobe period mutants and the regulatory spine (R-spine, purple) with F155 of the DFG motif in cyan. Polar interactions between Q51 and G156 that link the N- and C-lobe are depicted with a dashed black line. C, Alignment of the mammalian and Drosophila Degron and FASP/stabilizing sequences. Residues in bold have experimental support for phosphorylation. Dashed arrows indicate sequential phosphorylation following the consensus pSxxS motif.

Figure 7 Substrate discrimination on the PER2 phosphoswitch is regulated by the CK1 activation loop switch

A,B Western blot of FASP priming site, detecting pS659 (A) or Degron site, detecting pS478 (B) phosphorylation on mouse myc-PER2 in HEK293 cell lysates after transfection with indicated myc-CK1 $1 \varepsilon$ expression plasmids. 3M triple mutant: T44A, H46R, P47S. Representative blot from $n=3$ shown. C,D Western blot of FASP priming (C) or Degron (D) phosphorylation on PER2 as above with the myc-CK1\& K224D mutant. Representative blot from $\mathrm{n}=3$ shown with replicate samples loaded for tau and K224D. E, Overlaid ${ }^{15} \mathrm{~N} /{ }^{1} \mathrm{H}$ HSQC spectra at $3 \mathrm{hr}$ timepoint in the NMR kinase assay on $200 \mu \mathrm{M}{ }^{15} \mathrm{~N}$ FASP + $1 \mu \mathrm{M}$ K224D (teal) or WT (gray) CK1ס $\Delta \mathrm{C}$. Arrows, phosphospecific peaks corresponding to pS659, pS662, and pS665. F, Representative real-time luminescence data for PER2::LUC stability in HEK293 cells transfected with mycPER2::LUC plus empty vector (black) or myc-CK1є WT (gray) or tau (red) as indicated $(\mathrm{n}=4) .40 \mu \mathrm{g} / \mathrm{mL}$ cycloheximide $(\mathrm{CHX})$ added 24 hours post-transfection (arrow). $\mathrm{G}$, Quantification of PER2::LUC half-life with different myc-CK1 1 mutants. Data represent mean \pm s.d. $(n=4)$ with significance assessed as above. $H$, Scatterplot with linear

740 regression analysis of the ratio of enzyme efficiencies $\left(k_{\mathrm{cat}} / K_{\mathrm{m}}\right)$ for FASP and Degron relative to the PER2::LUC half-life determined in panel $G$ and Figure S7. All data are plotted as mean \pm s.d. ( $n=4$ for PER2::LUC and $n=3-4$ for enzyme efficiencies). Black, linear regression to data; gray, 95\% confidence interval. I, The conformational switch of the CK1 $\delta / \varepsilon$ activation loop is coupled to substrate selection in the PER2

745 phosphoswitch. Left, the activation loop of the WT kinase is stable in the 'loop down' conformation, leading to preferential phosphorylation the FASP region, which stabilizes PER2 by reducing phosphorylation of the Degron. Right, the activation loop of tau kinase is better tolerated in the alternate 'loop up' conformation leading to an intrinsic gain of kinase function on the Degron and loss of kinase function on the stabilizing

750 FASP region. This switch in substrate preference promotes PER2 degradation and leads to a shorter circadian period. CKBD, CK1 binding domain; CBD, CRY binding domain. See also Figure S7.

\section{References Cited}

755 Abe, J., Hiyama, T.B., Mukaiyama, A., Son, S., Mori, T., Saito, S., Osako, M., Wolanin, J., Yamashita, E., Kondo, T., et al. (2015). Circadian rhythms. Atomic-scale origins of slowness in the cyanobacterial circadian clock. Science 349, 312-316. 
Aryal, R.P., Kwak, P.B., Tamayo, A.G., Gebert, M., Chiu, P.L., Walz, T., and Weitz, C.J. (2017). Macromolecular Assemblies of the Mammalian Circadian Clock. Molecular cell 67, 770-782 e776.

Balsalobre, A., Marcacci, L., and Schibler, U. (2000). Multiple signaling pathways elicit circadian gene expression in cultured Rat-1 fibroblasts. Current biology : CB 10, 12911294.

Bass, J., and Lazar, M.A. (2016). Circadian time signatures of fitness and disease. Science 354, 994-999.

770 Chen, R., Schirmer, A., Lee, Y., Lee, H., Kumar, V., Yoo, S.H., Takahashi, J.S., and Lee, C. (2009). Rhythmic PER abundance defines a critical nodal point for negative feedback within the circadian clock mechanism. Molecular cell 36, 417-430.

Chiu, J.C., Vanselow, J.T., Kramer, A., and Edery, I. (2008). The phospho-occupancy of an atypical SLIMB-binding site on PERIOD that is phosphorylated by DOUBLETIME controls the pace of the clock. Genes \& development 22, 1758-1772.

Eide, E.J., Woolf, M.F., Kang, H., Woolf, P., Hurst, W., Camacho, F., Vielhaber, E.L., Giovanni, A., and Virshup, D.M. (2005). Control of mammalian circadian rhythm by CKlepsilon-regulated proteasome-mediated PER2 degradation. Molecular and cellular biology 25, 2795-2807.

Eng, G.W.L., Edison, and Virshup, D.M. (2017). Site-specific phosphorylation of casein kinase 1 delta (CK1delta) regulates its activity towards the circadian regulator PER2.

785 PloS one 12, e0177834.

Fan, J.Y., Preuss, F., Muskus, M.J., Bjes, E.S., and Price, J.L. (2009). Drosophila and vertebrate casein kinase Idelta exhibits evolutionary conservation of circadian function. Genetics 181, 139-152.

Flotow, H., Graves, P.R., Wang, A.Q., Fiol, C.J., Roeske, R.W., and Roach, P.J. (1990). Phosphate groups as substrate determinants for casein kinase I action. The Journal of biological chemistry 265, 14264-14269.

795 Fustin, J.M., Kojima, R., Itoh, K., Chang, H.Y., Ye, S., Zhuang, B., Oji, A., Gibo, S., Narasimamurthy, R., Virshup, D., et al. (2018). Two Ck1delta transcripts regulated by m6A methylation code for two antagonistic kinases in the control of the circadian clock. Proceedings of the National Academy of Sciences of the United States of America 115, 5980-5985.

Gallego, M., Eide, E.J., Woolf, M.F., Virshup, D.M., and Forger, D.B. (2006). An opposite role for tau in circadian rhythms revealed by mathematical modeling. 
Proceedings of the National Academy of Sciences of the United States of America 103, 10618-10623.

Gibbs, C.S., and Zoller, M.J. (1991). Rational scanning mutagenesis of a protein kinase identifies functional regions involved in catalysis and substrate interactions. The Journal of biological chemistry 266, 8923-8931.

810 Goldsmith, E.J., Akella, R., Min, X., Zhou, T., and Humphreys, J.M. (2007). Substrate and docking interactions in serine/threonine protein kinases. Chem Rev 107, 50655081.

Gorl, M., Merrow, M., Huttner, B., Johnson, J., Roenneberg, T., and Brunner, M. (2001). A PEST-like element in FREQUENCY determines the length of the circadian period in Neurospora crassa. EMBO J 20, 7074-7084.

Graves, P.R., and Roach, P.J. (1995). Role of COOH-terminal phosphorylation in the regulation of casein kinase I delta. The Journal of biological chemistry 270,21689 21694.

Hayasaka, N., Hirano, A., Miyoshi, Y., Tokuda, I.T., Yoshitane, H., Matsuda, J., and Fukada, Y. (2017). Salt-inducible kinase 3 regulates the mammalian circadian clock by destabilizing PER2 protein. Elife 6.

Hirano, A., Fu, Y.H., and Ptacek, L.J. (2016). The intricate dance of post-translational modifications in the rhythm of life. Nat Struct Mol Biol 23, 1053-1060.

Hirota, T., and Kay, S.A. (2009). High-throughput screening and chemical biology: new approaches for understanding circadian clock mechanisms. Chem Biol 16, 921-927.

Isojima, Y., Nakajima, M., Ukai, H., Fujishima, H., Yamada, R.G., Masumoto, K.H., Kiuchi, R., Ishida, M., Ukai-Tadenuma, M., Minami, Y., et al. (2009). CKlepsilon/deltadependent phosphorylation is a temperature-insensitive, period-determining process in the mammalian circadian clock. Proceedings of the National Academy of Sciences of the United States of America 106, 15744-15749.

Johnson, L.N., Noble, M.E., and Owen, D.J. (1996). Active and inactive protein kinases: structural basis for regulation. Cell 85, 149-158.

Jones, C.R., Huang, A.L., Ptacek, L.J., and Fu, Y.H. (2013). Genetic basis of human circadian rhythm disorders. Exp Neurol 243, 28-33.

Kawakami, F., Suzuki, K., and Ohtsuki, K. (2008). A novel consensus phosphorylation 845 motif in sulfatide- and cholesterol-3-sulfate-binding protein substrates for CK1 in vitro. Biol Pharm Bull 31, 193-200. 
Kim, J.K., and Forger, D.B. (2012). A mechanism for robust circadian timekeeping via stoichiometric balance. Mol Syst Biol 8, 630.

Kivimae, S., Saez, L., and Young, M.W. (2008). Activating PER repressor through a DBT-directed phosphorylation switch. PLoS biology 6, e183.

Kloss, B., Price, J.L., Saez, L., Blau, J., Rothenfluh, A., Wesley, C.S., and Young, M.W. (1998). The Drosophila clock gene double-time encodes a protein closely related to human casein kinase lepsilon. Cell 94, 97-107.

Knippschild, U., Milne, D.M., Campbell, L.E., DeMaggio, A.J., Christenson, E., Hoekstra, M.F., and Meek, D.W. (1997). p53 is phosphorylated in vitro and in vivo by the delta and epsilon isoforms of casein kinase 1 and enhances the level of casein kinase 1 delta in response to topoisomerase-directed drugs. Oncogene 15, 1727-1736.

Kojima, S., Shingle, D.L., and Green, C.B. (2011). Post-transcriptional control of circadian rhythms. J Cell Sci 124, 311-320.

Kornev, A.P., and Taylor, S.S. (2015). Dynamics-Driven Allostery in Protein Kinases. Trends Biochem Sci 40, 628-647.

Lee, C., Etchegaray, J.P., Cagampang, F.R., Loudon, A.S., and Reppert, S.M. (2001). Posttranslational mechanisms regulate the mammalian circadian clock. Cell 107, 855867.

Lee, C., Weaver, D.R., and Reppert, S.M. (2004). Direct association between mouse PERIOD and CKlepsilon is critical for a functioning circadian clock. Molecular and 875 cellular biology 24, 584-594.

Lee, H.M., Chen, R., Kim, H., Etchegaray, J.P., Weaver, D.R., and Lee, C. (2011a). The period of the circadian oscillator is primarily determined by the balance between casein kinase 1 and protein phosphatase 1. Proceedings of the National Academy of Sciences of the United States of America 108, 16451-16456.

Lee, Y., Chen, R., Lee, H.M., and Lee, C. (2011b). Stoichiometric relationship among clock proteins determines robustness of circadian rhythms. The Journal of biological chemistry 286, 7033-7042.

Leon, B.C., Tsigelny, I., and Adams, J.A. (2001). Electrostatic environment surrounding the activation loop phosphotyrosine in the oncoprotein v-Fps. Biochemistry 40, 1007810086.

890 Long, A.M., Zhao, H., and Huang, X. (2012). Structural basis for the potent and selective inhibition of casein kinase 1 epsilon. J Med Chem 55, 10307-10311. 
Longenecker, K.L., Roach, P.J., and Hurley, T.D. (1996). Three-dimensional structure of mammalian casein kinase I: molecular basis for phosphate recognition. Journal of molecular biology 257, 618-631.

Lowrey, P.L., Shimomura, K., Antoch, M.P., Yamazaki, S., Zemenides, P.D., Ralph, M.R., Menaker, M., and Takahashi, J.S. (2000). Positional syntenic cloning and functional characterization of the mammalian circadian mutation tau. Science $288,483-$ 492.

Marin, O., Bustos, V.H., Cesaro, L., Meggio, F., Pagano, M.A., Antonelli, M., Allende, C.C., Pinna, L.A., and Allende, J.E. (2003). A noncanonical sequence phosphorylated by casein kinase 1 in beta-catenin may play a role in casein kinase 1 targeting of important signaling proteins. Proceedings of the National Academy of Sciences of the United States of America 100, 10193-10200.

Marin, O., Meggio, F., and Pinna, L.A. (1994). Design and synthesis of two new peptide substrates for the specific and sensitive monitoring of casein kinases-1 and -2 .

910 Biochemical and biophysical research communications 198, 898-905.

McClendon, C.L., Kornev, A.P., Gilson, M.K., and Taylor, S.S. (2014). Dynamic architecture of a protein kinase. Proceedings of the National Academy of Sciences of the United States of America 111, E4623-4631.

Miao, Y., Feher, V.A., and McCammon, J.A. (2015). Gaussian Accelerated Molecular Dynamics: Unconstrained Enhanced Sampling and Free Energy Calculation. J Chem Theory Comput 11, 3584-3595.

920 Michael, A.K., Fribourgh, J.L., Chelliah, Y., Sandate, C.R., Hura, G.L., SchneidmanDuhovny, D., Tripathi, S.M., Takahashi, J.S., and Partch, C.L. (2017). Formation of a repressive complex in the mammalian circadian clock is mediated by the secondary pocket of CRY1. Proceedings of the National Academy of Sciences of the United States of America 114, 1560-1565.

Minzel, W., Venkatachalam, A., Fink, A., Hung, E., Brachya, G., Burstain, I., Shaham, M., Rivlin, A., Omer, I., Zinger, A., et al. (2018). Small Molecules Co-targeting CKIalpha and the Transcriptional Kinases CDK7/9 Control AML in Preclinical Models. Cell 175, 171-185 e125.

Muskus, M.J., Preuss, F., Fan, J.Y., Bjes, E.S., and Price, J.L. (2007). Drosophila DBT lacking protein kinase activity produces long-period and arrhythmic circadian behavioral and molecular rhythms. Molecular and cellular biology 27, 8049-8064.

935 Narasimamurthy, R., Hunt, S.R., Lu, Y., Fustin, J.M., Okamura, H., Partch, C.L., Forger, D.B., Kim, J.K., and Virshup, D.M. (2018). CK1delta/epsilon protein kinase primes the PER2 circadian phosphoswitch. Proceedings of the National Academy of Sciences of the United States of America 115, 5986-5991. 
940 Nolen, B., Taylor, S., and Ghosh, G. (2004). Regulation of protein kinases; controlling activity through activation segment conformation. Molecular cell 15, 661-675.

Oshima, T., Niwa, Y., Kuwata, K., Srivastava, A., Hyoda, T., Tsuchiya, Y., Kumagai, M., Tsuyuguchi, M., Tamaru, T., Sugiyama, A., et al. (2019). Cell-based screen identifies a new potent and highly selective CK2 inhibitor for modulation of circadian rhythms and cancer cell growth. Sci Adv 5, eaau9060.

Penas, C., Govek, E.E., Fang, Y., Ramachandran, V., Daniel, M., Wang, W., Maloof, M.E., Rahaim, R.J., Bibian, M., Kawauchi, D., et al. (2015). Casein kinase 1delta is an $\mathrm{APC} / \mathrm{C}(\mathrm{Cdh} 1)$ substrate that regulates cerebellar granule cell neurogenesis. Cell Rep $11,249-260$.

Price, J.L., Blau, J., Rothenfluh, A., Abodeely, M., Kloss, B., and Young, M.W. (1998). double-time is a novel Drosophila clock gene that regulates PERIOD protein accumulation. Cell 94, 83-95.

Qin, X., Mori, T., Zhang, Y., and Johnson, C.H. (2015). PER2 Differentially Regulates Clock Phosphorylation versus Transcription by Reciprocal Switching of CK1epsilon Activity. Journal of biological rhythms 30, 206-216.

Ralph, M.R., and Menaker, M. (1988). A mutation of the circadian system in golden hamsters. Science 241, 1225-1227.

Reischl, S., Vanselow, K., Westermark, P.O., Thierfelder, N., Maier, B., Herzel, H., and Kramer, A. (2007). Beta-TrCP1-mediated degradation of PERIOD2 is essential for circadian dynamics. Journal of biological rhythms 22, 375-386.

Rivers, A., Gietzen, K.F., Vielhaber, E., and Virshup, D.M. (1998). Regulation of casein kinase I epsilon and casein kinase I delta by an in vivo futile phosphorylation cycle. The Journal of biological chemistry 273, 15980-15984.

Rothenfluh, A., Abodeely, M., and Young, M.W. (2000). Short-period mutations of per affect a double-time-dependent step in the Drosophila circadian clock. Current biology : CB 10, 1399-1402.

Shinohara, Y., Koyama, Y.M., Ukai-Tadenuma, M., Hirokawa, T., Kikuchi, M., Yamada, R.G., Ukai, H., Fujishima, H., Umehara, T., Tainaka, K., et al. (2017). TemperatureSensitive Substrate and Product Binding Underlie Temperature-Compensated Phosphorylation in the Clock. Molecular cell 67, 783-798 e720.

Skamnaki, V.T., Owen, D.J., Noble, M.E., Lowe, E.D., Lowe, G., Oikonomakos, N.G., and Johnson, L.N. (1999). Catalytic mechanism of phosphorylase kinase probed by mutational studies. Biochemistry 38, 14718-14730. 
985 Suri, V., Hall, J.C., and Rosbash, M. (2000). Two novel doubletime mutants alter circadian properties and eliminate the delay between RNA and protein in Drosophila. The Journal of neuroscience : the official journal of the Society for Neuroscience 20, 7547-7555.

990 Takahashi, J.S. (2017). Transcriptional architecture of the mammalian circadian clock. Nature reviews Genetics 18, 164-179.

Taylor, S.S., and Kornev, A.P. (2011). Protein kinases: evolution of dynamic regulatory proteins. Trends Biochem Sci 36, 65-77.

Theillet, F.X., Rose, H.M., Liokatis, S., Binolfi, A., Thongwichian, R., Stuiver, M., and Selenko, P. (2013). Site-specific NMR mapping and time-resolved monitoring of serine and threonine phosphorylation in reconstituted kinase reactions and mammalian cell extracts. Nat Protoc 8, 1416-1432.

Toh, K.L., Jones, C.R., He, Y., Eide, E.J., Hinz, W.A., Virshup, D.M., Ptacek, L.J., and Fu, Y.H. (2001). An hPer2 phosphorylation site mutation in familial advanced sleep phase syndrome. Science 291, 1040-1043.

1005 Top, D., O'Neil, J.L., Merz, G.E., Dusad, K., Crane, B.R., and Young, M.W. (2018). CK1/Doubletime activity delays transcription activation in the circadian clock. Elife 7.

van Ooijen, G., Hindle, M., Martin, S.F., Barrios-Llerena, M., Sanchez, F., Bouget, F.Y., O'Neill, J.S., Le Bihan, T., and Millar, A.J. (2013). Functional analysis of Casein Kinase 10101 in a minimal circadian system. PloS one 8, e70021.

Vanselow, K., Vanselow, J.T., Westermark, P.O., Reischl, S., Maier, B., Korte, T., Herrmann, A., Herzel, H., Schlosser, A., and Kramer, A. (2006). Differential effects of PER2 phosphorylation: molecular basis for the human familial advanced sleep phase 1015 syndrome (FASPS). Genes \& development 20, 2660-2672.

Venerando, A., Ruzzene, M., and Pinna, L.A. (2014). Casein kinase: the triple meaning of a misnomer. Biochem J 460, 141-156.

1020 Venkatesan, A., Fan, J.Y., Bouyain, S., and Price, J.L. (2019). The Circadian tau Mutation in Casein Kinase 1 Is Part of a Larger Domain That Can Be Mutated to Shorten Circadian Period. Int J Mol Sci 20.

Xu, H., Gustafson, C.L., Sammons, P.J., Khan, S.K., Parsley, N.C., Ramanathan, C., 1025 Lee, H.W., Liu, A.C., and Partch, C.L. (2015). Cryptochrome 1 regulates the circadian clock through dynamic interactions with the BMAL1 C terminus. Nat Struct Mol Biol 22, 476-484. 
Xu, Y., Padiath, Q.S., Shapiro, R.E., Jones, C.R., Wu, S.C., Saigoh, N., Saigoh, K., 1030 Ptacek, L.J., and Fu, Y.H. (2005). Functional consequences of a CKIdelta mutation causing familial advanced sleep phase syndrome. Nature 434, 640-644.

Xu, Y., Toh, K.L., Jones, C.R., Shin, J.Y., Fu, Y.H., and Ptacek, L.J. (2007). Modeling of a human circadian mutation yields insights into clock regulation by PER2. Cell 128, 59-

103570.

Yu, W., Zheng, H., Houl, J.H., Dauwalder, B., and Hardin, P.E. (2006). PER-dependent rhythms in CLK phosphorylation and E-box binding regulate circadian transcription. Genes \& development 20, 723-733.

1040

Zeringo, N.A., and Bellizzi, J.J., 3rd (2014). A PER2-derived mechanism-based bisubstrate analog for casein kinase 1epsilon. Chem Biol Drug Des 84, 697-703.

Zhou, M., Kim, J.K., Eng, G.W., Forger, D.B., and Virshup, D.M. (2015). A Period2

1045 Phosphoswitch Regulates and Temperature Compensates Circadian Period. Molecular cell $60,77-88$.

Zilian, O., Frei, E., Burke, R., Brentrup, D., Gutjahr, T., Bryant, P.J., and Noll, M. (1999). double-time is identical to discs overgrown, which is required for cell survival,

1050 proliferation and growth arrest in Drosophila imaginal discs. Development 126, 54095420.

Zylka, M.J., Shearman, L.P., Weaver, D.R., and Reppert, S.M. (1998). Three period homologs in mammals: differential light responses in the suprachiasmatic circadian 1055 clock and oscillating transcripts outside of brain. Neuron 20, 1103-1110. 
A

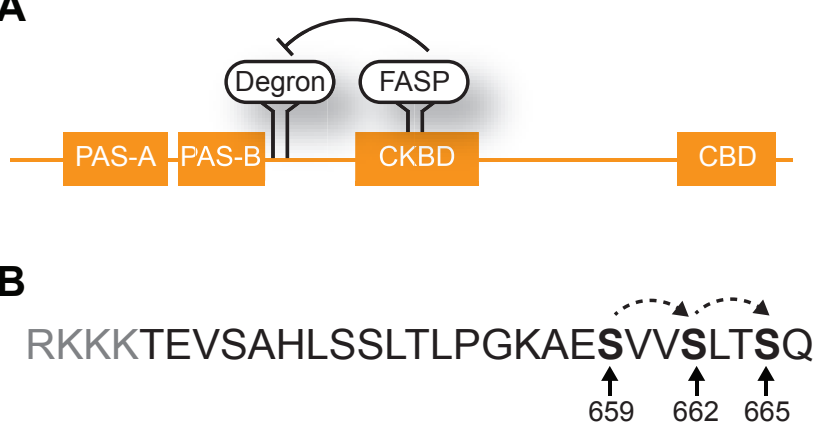

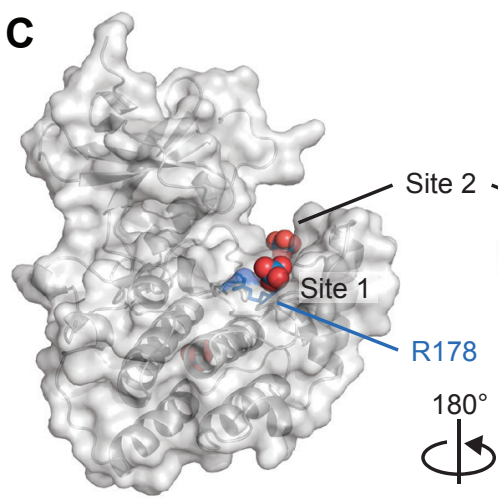

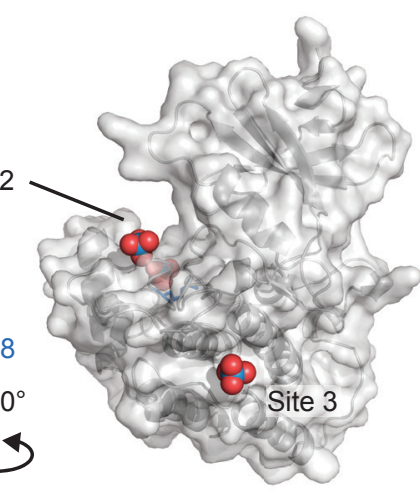

D

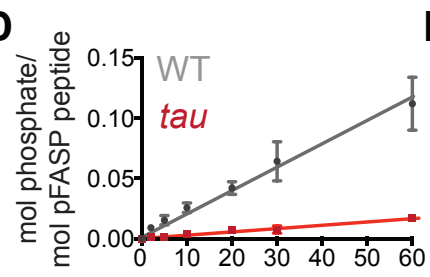

E

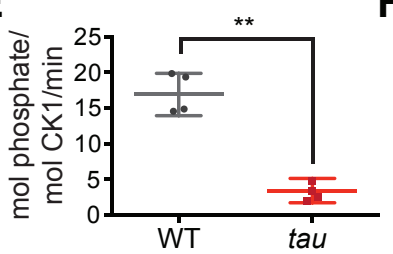

F
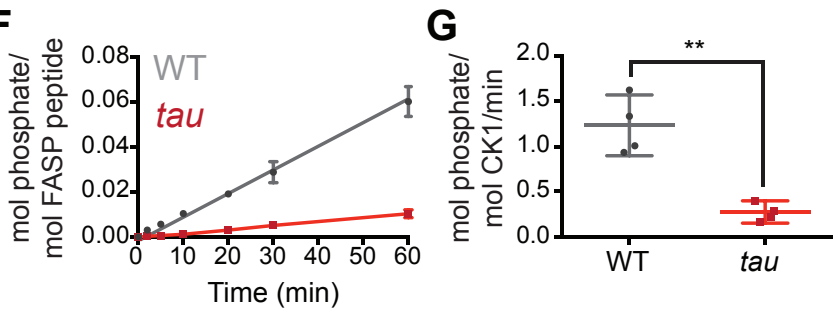

H

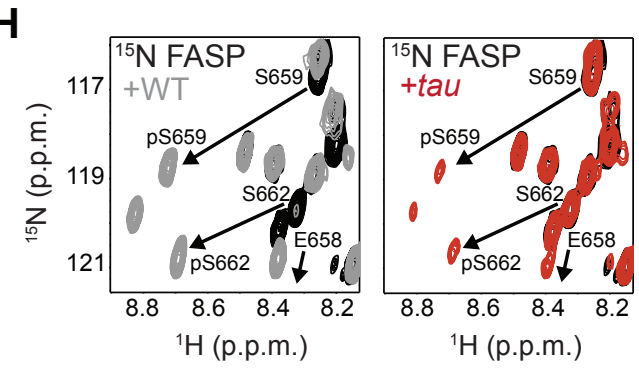

K

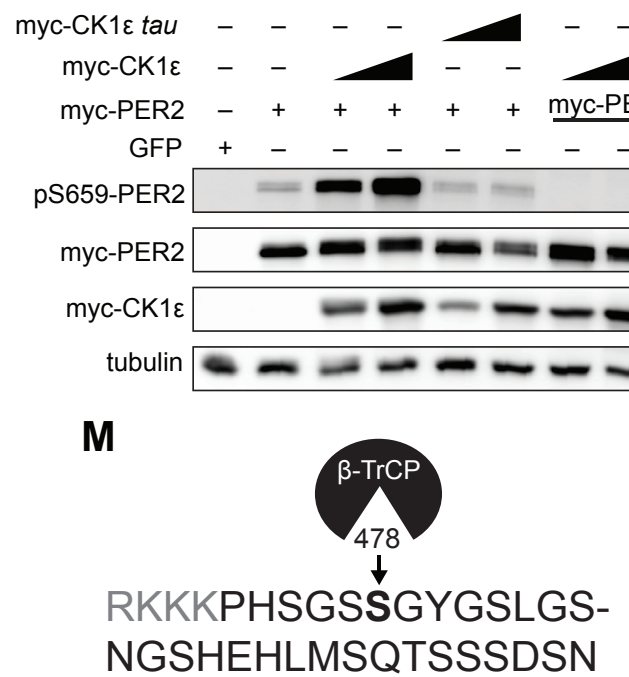

I

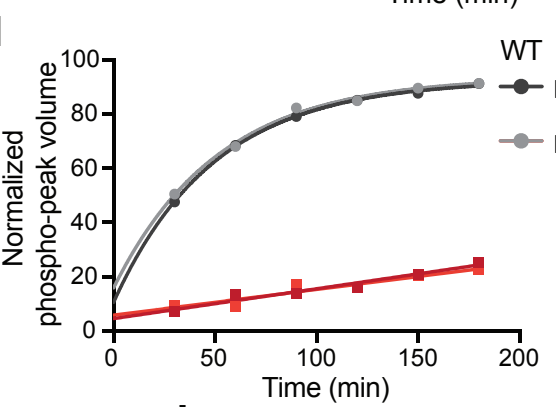

L

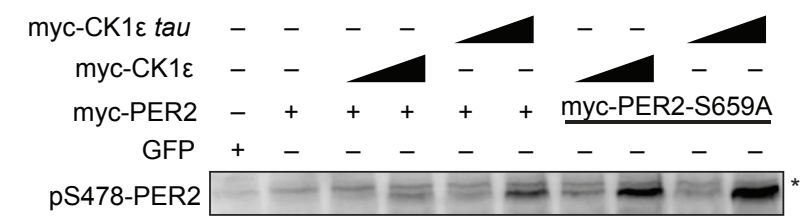

myc-PER2

(2)

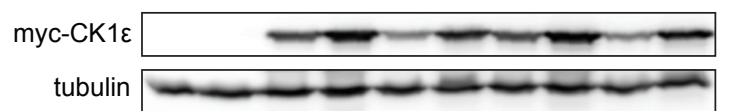

0

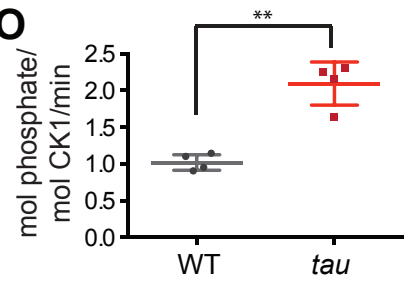



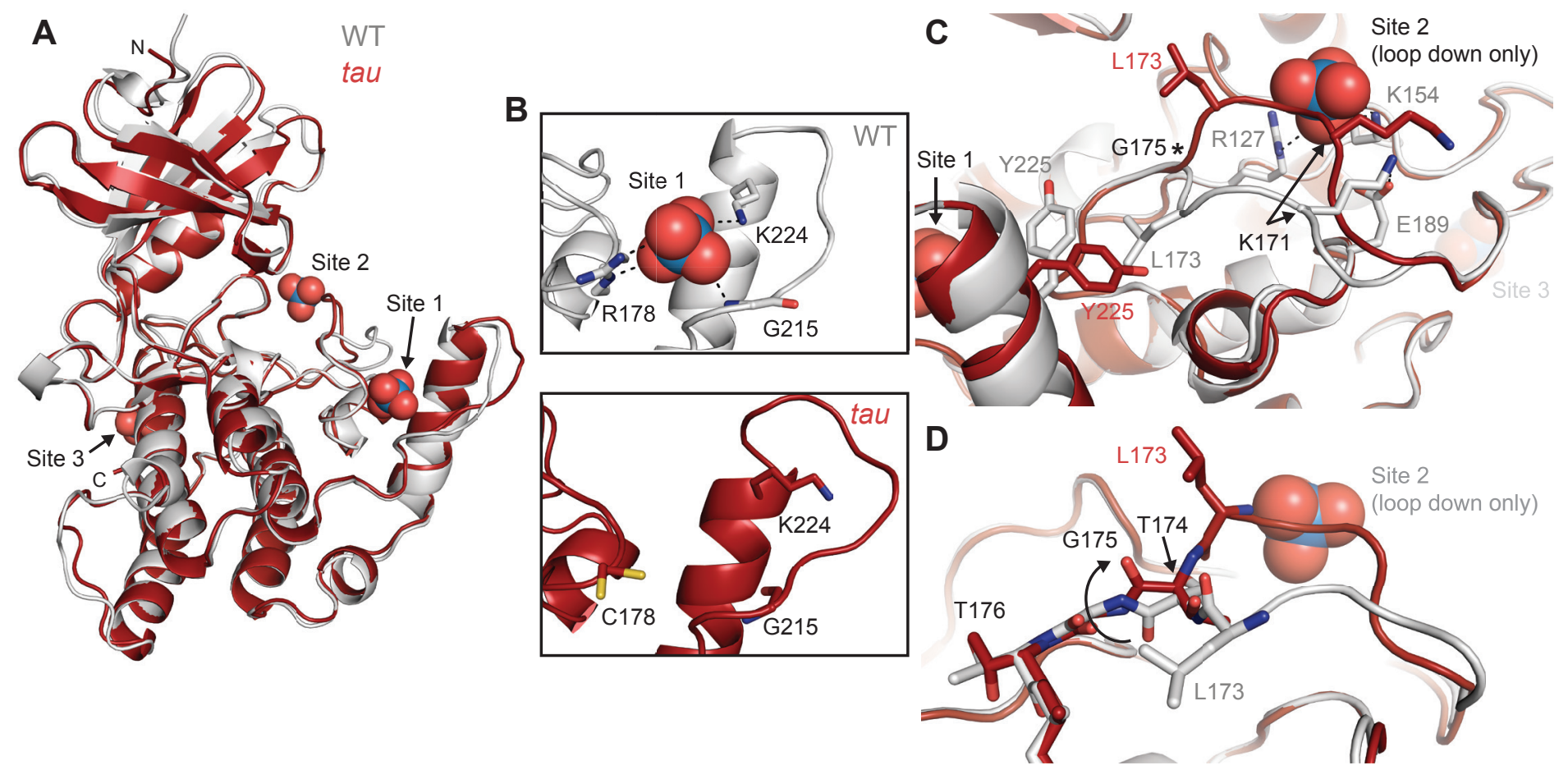

E

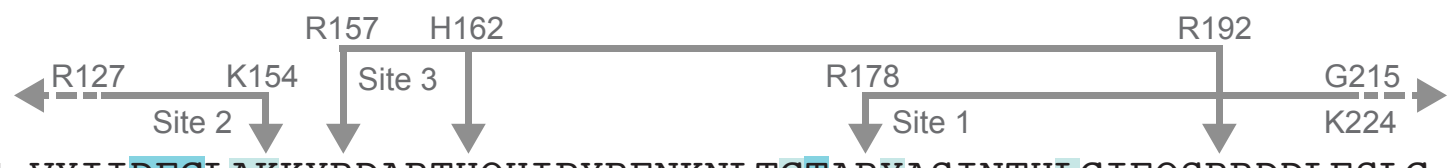

(CK1) CK1 VYIIDFGLAKKYRDARTHQHIPYRENKNLTGTARYASINTHLGIEQSRRDDLESLG

(STE) TAO2 VKLGDFGSAS IMAPAN----------SFVGTPYWMAPEVILAMDEGQYDGKVDVW

(CMGC) CDK2 IKLADFGLARAFGVPV-------RTYTHEVVTLWYRAPEILLG--CKYYSTAVDIW

(AGC) PKA IQVTDFGFAKRVKG--------RTWT-LCGTPEYLAPEIIL---SKGYNKAVDDW

(CAMK) CAMK IMISDFGLSKMEDPGS-------VLST-ACGTPGYVAPEVLA---QKPYSKAVDCW

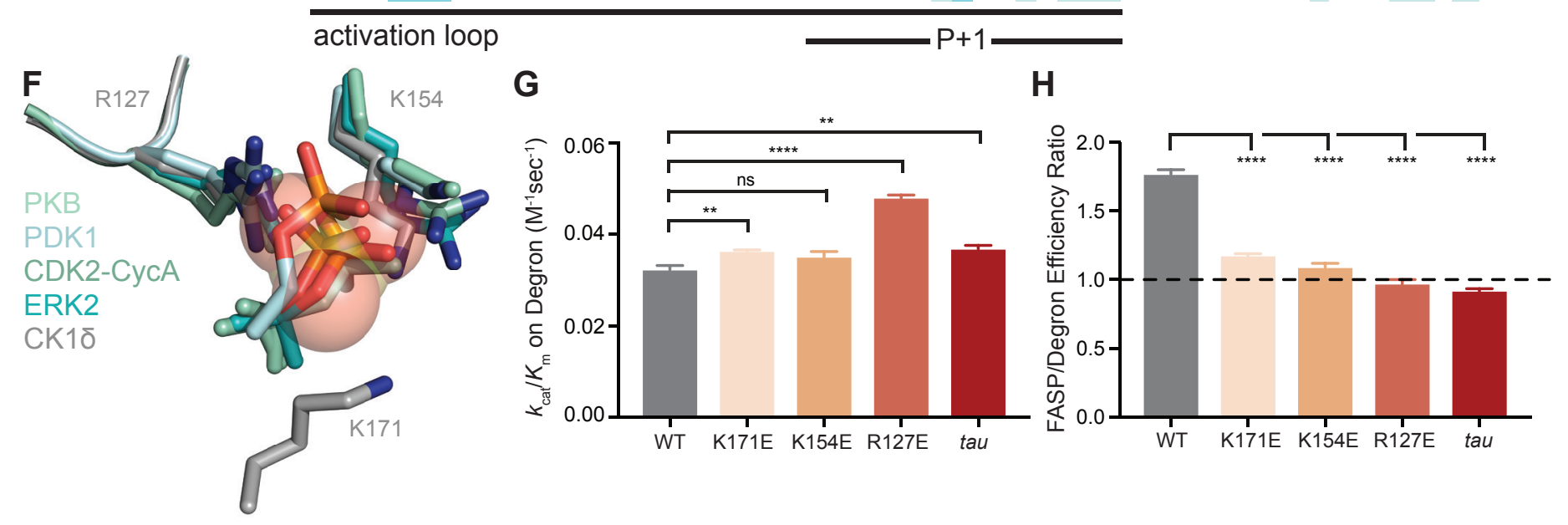

Figure 2 

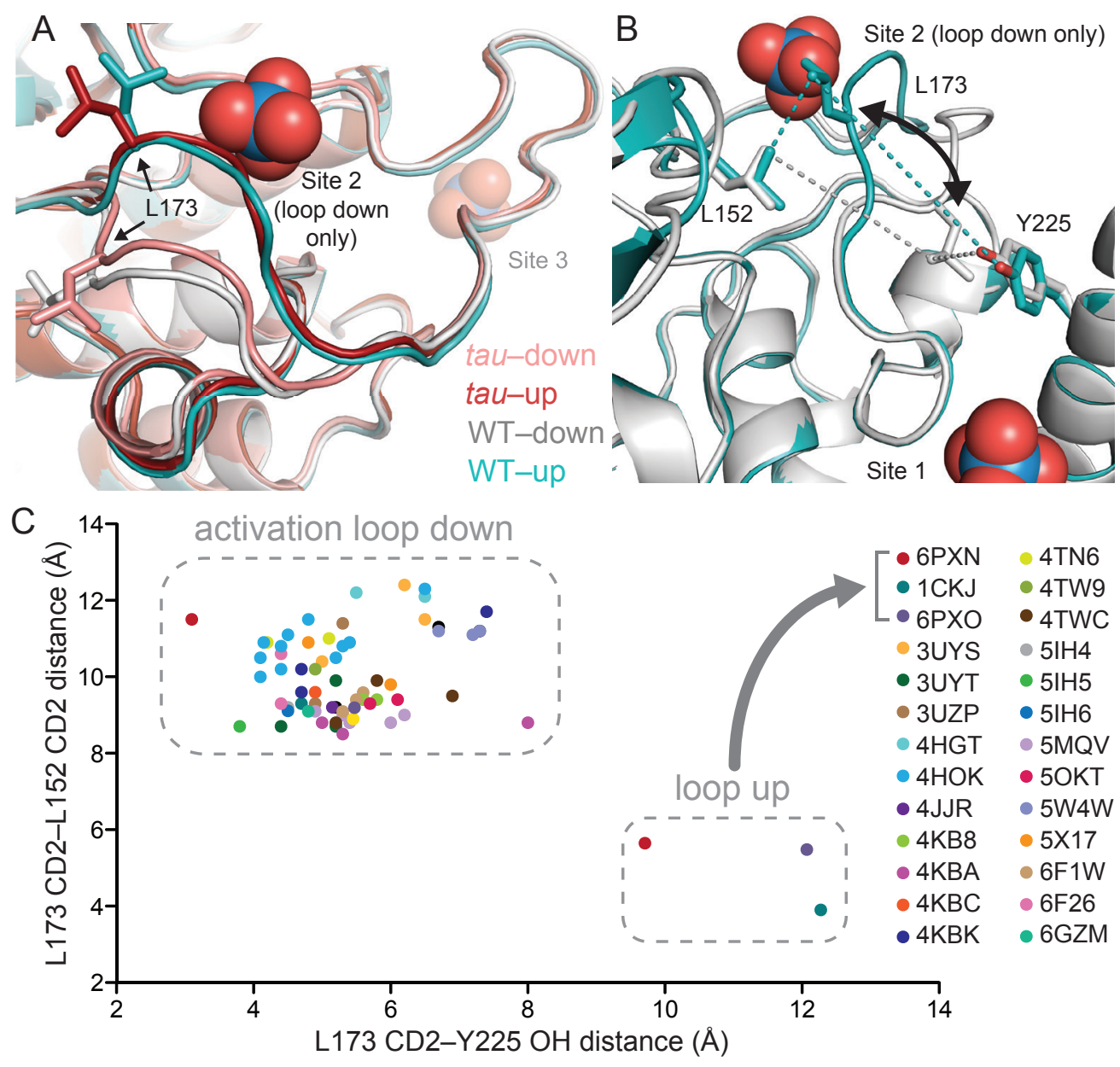
bioRxiv preprint doi: https://doi.org/10.1101/734624; this version posted August 14,2019 . The copyright holder for this preprint (which was not certified by peer review) is the author/funder. All rights reserved. No reuse allowed without permission.

A

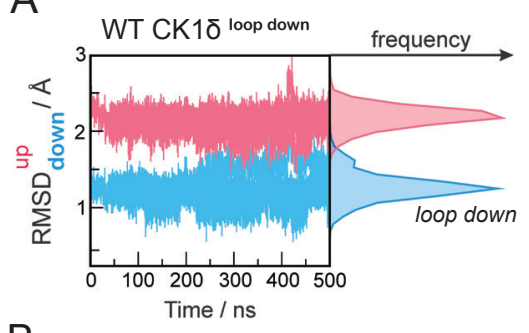

B

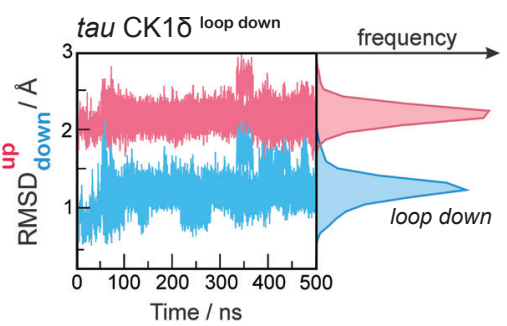

C

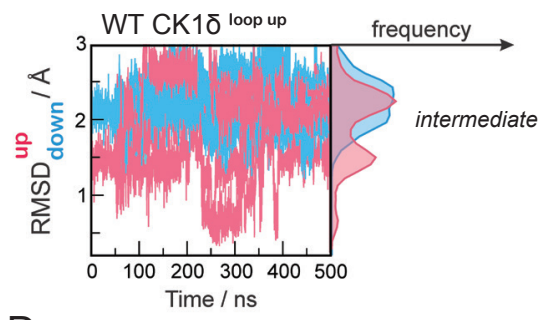

D

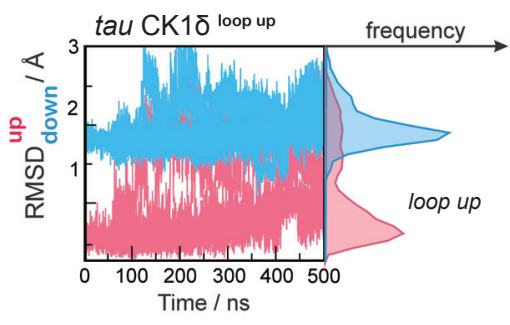

E
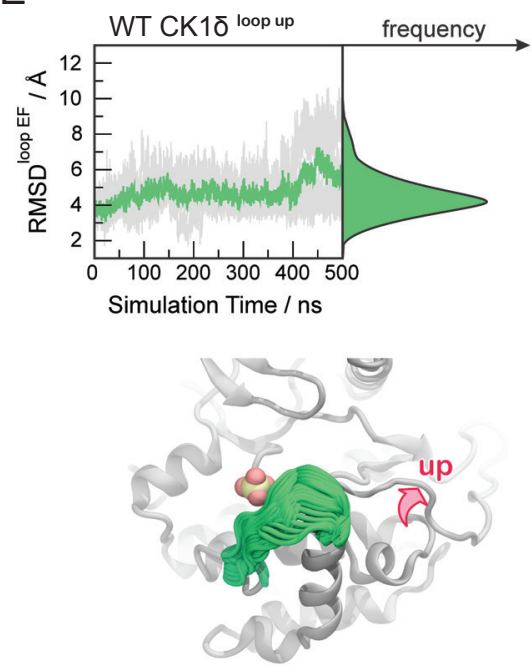

G
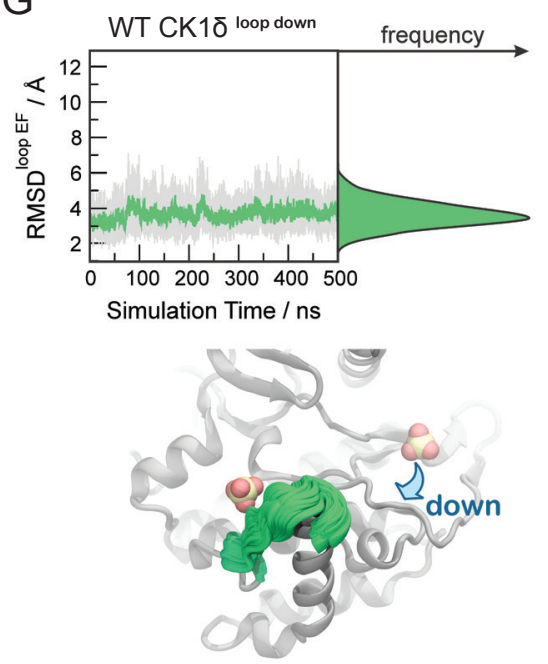

F
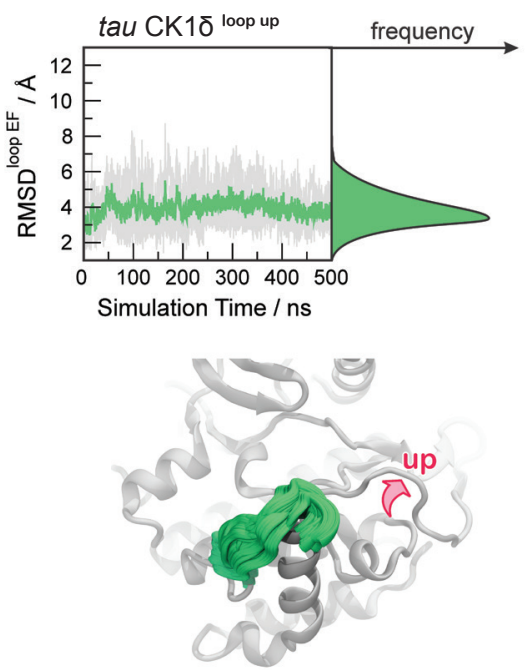

$\mathrm{H}$

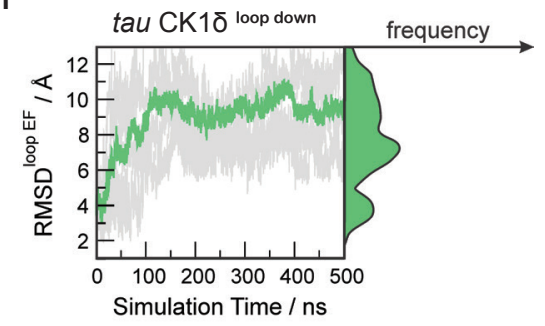

I
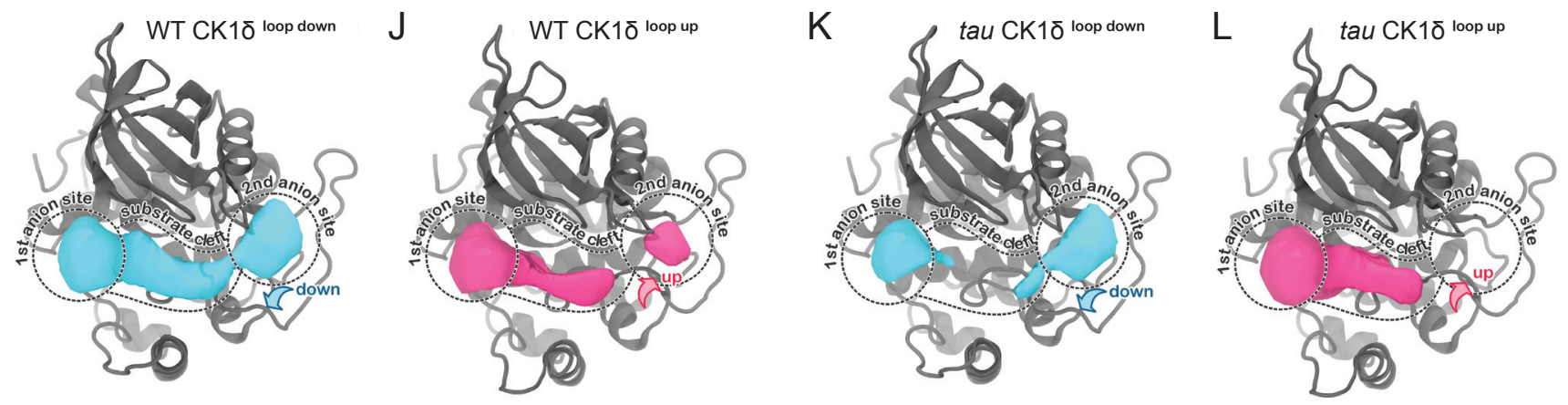

Figure 4 
A

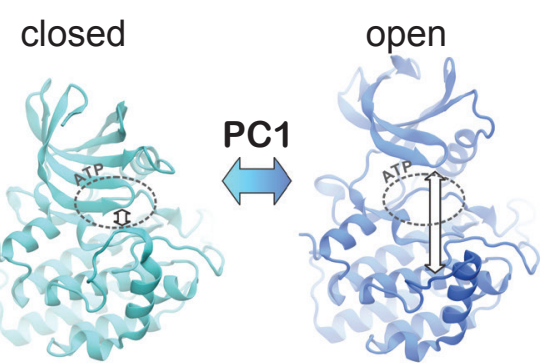

C

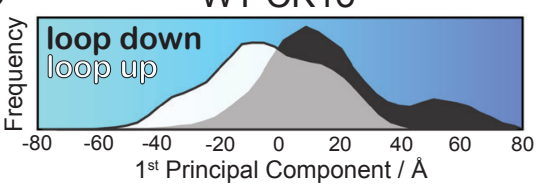

tau CK1ס

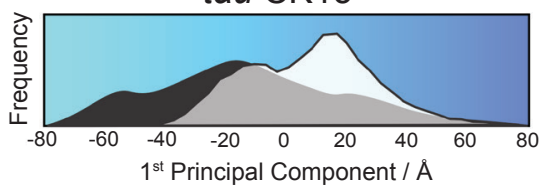

B collapsed extended

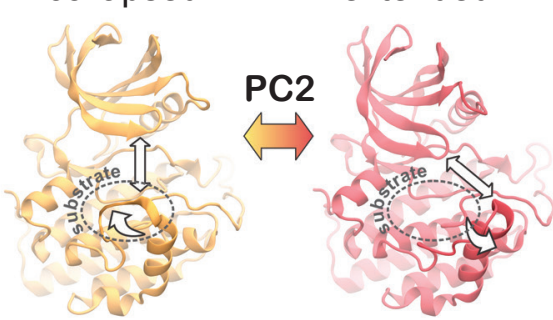

WT CK1ס

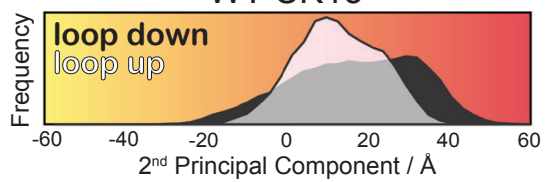

tau CK1ס

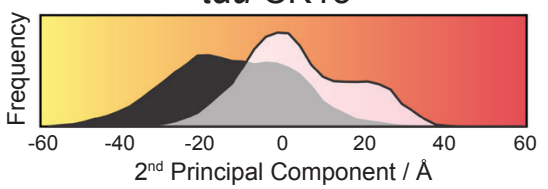

Figure 5 
A

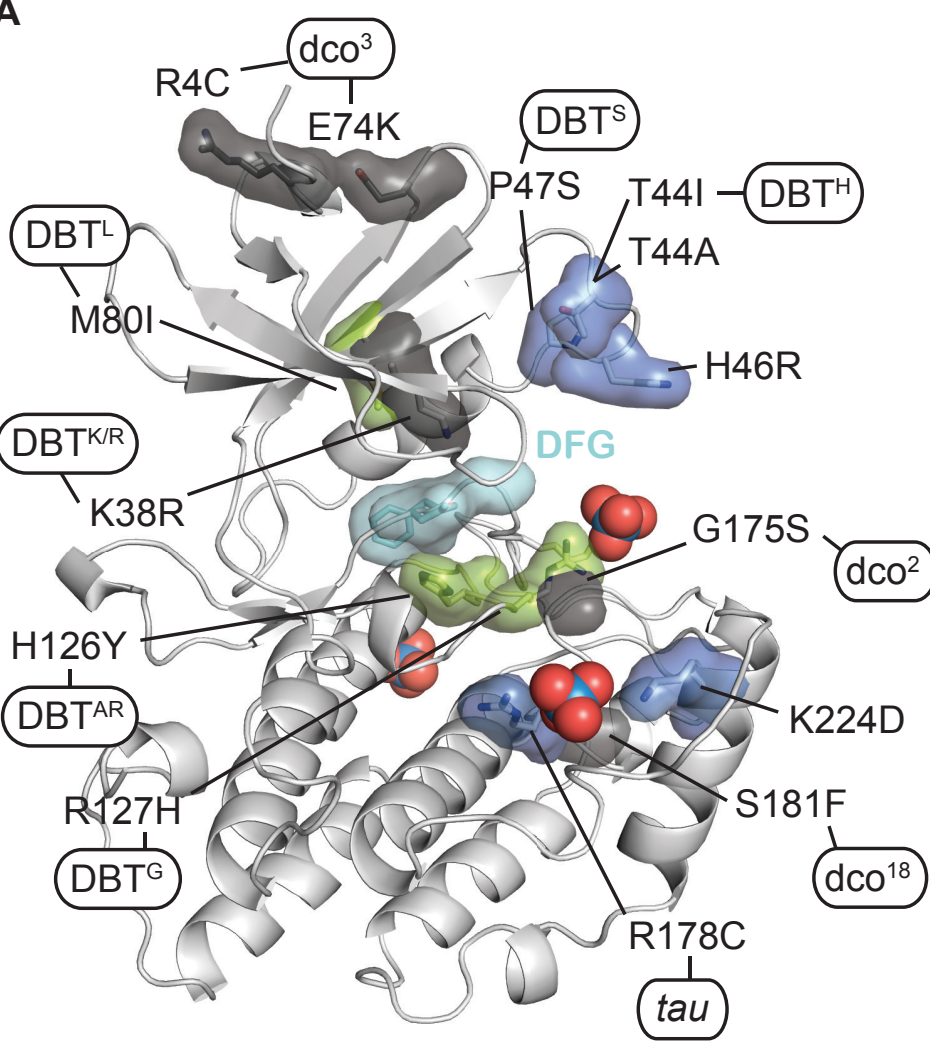

\section{short period}

\section{long period}

C

Degron

478 (mouse)

Mouse PHSGSSGYGLGSNGSHEH

Human PHSGSSGYGSLGSNGSHEH

Drosophila HSSGSSGYGGKPSTQASSS

$\stackrel{4}{4}$

loss of function

FASP
B

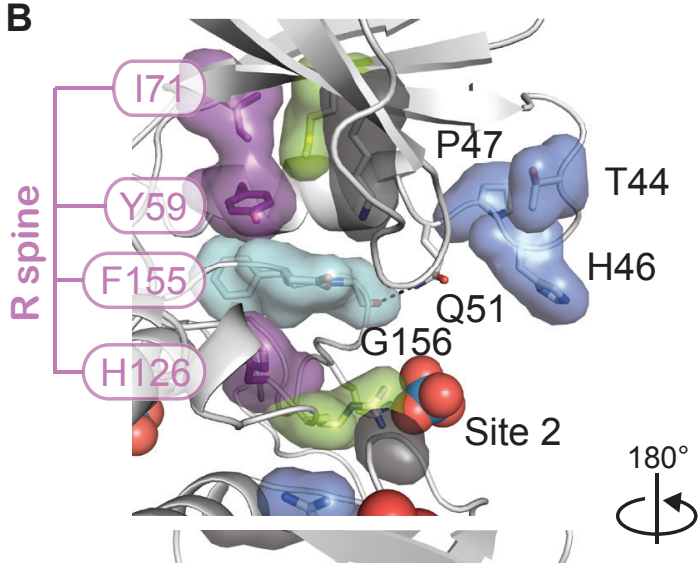

Mouse SLTLPGKAESVVSLTSQCSYSTSIVHVGDKK

Human SLALPGKAESVASLTSOCSYSSTIVHVGDKK

Drosophila NELTVSERDSVMLGEISPHHDYYDSKSSTET 4
589 
A

C

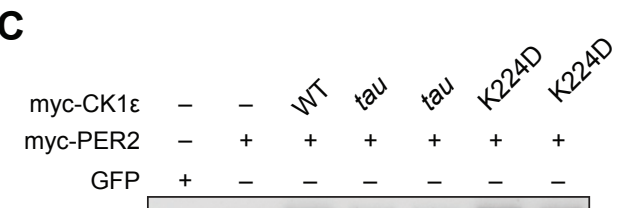

B

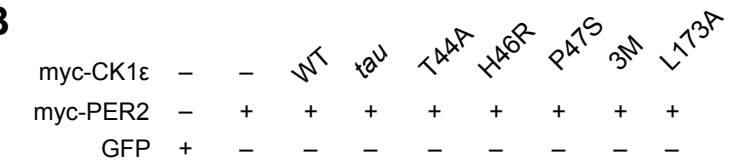

pS478-PER2

myc-PER2

myc-CK $1 \varepsilon$

tubulin

D

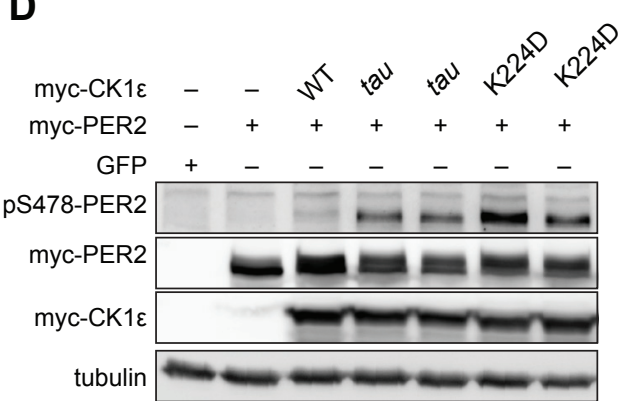

E

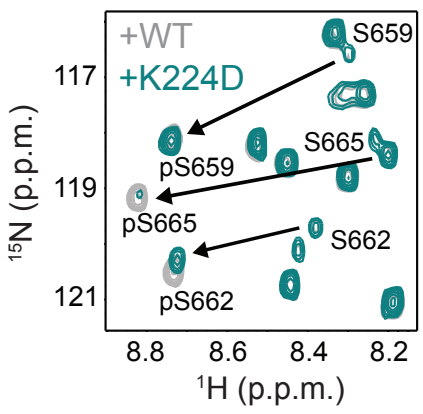

$\mathbf{F}$

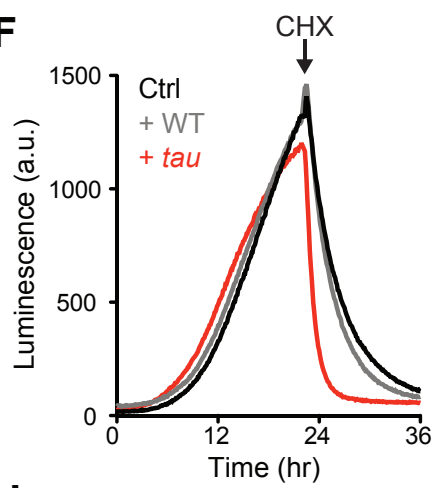

I

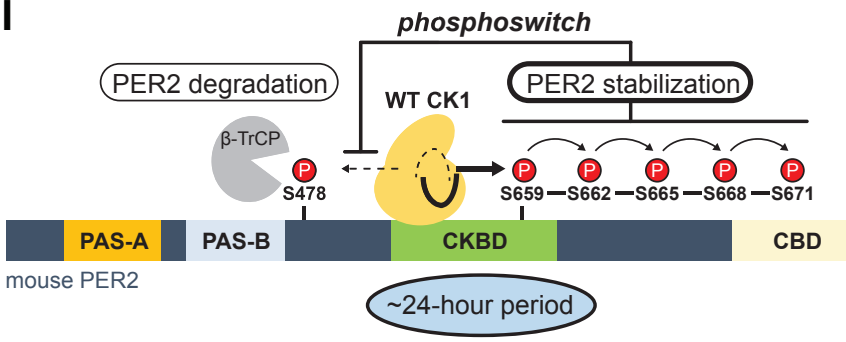

G

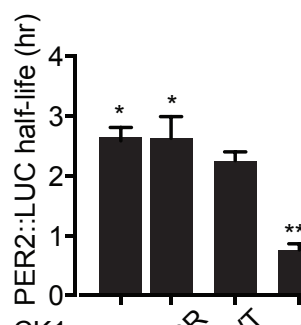

myc-CK1ع:

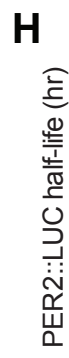

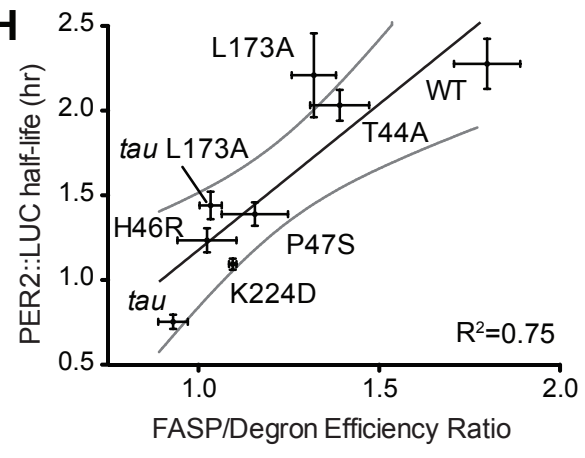

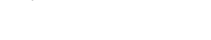

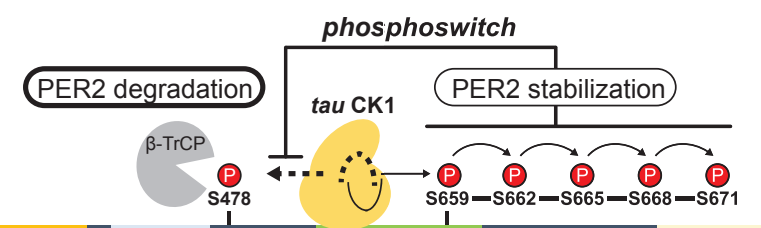

PAS-A PAS-B CKBD CBD

-20-hour period 